\title{
Loss of furin cleavage site attenuates SARS-CoV-2 pathogenesis
}

https://doi.org/10.1038/s41586-021-03237-4

Received: 26 August 2020

Accepted: 13 January 2021

Published online: 25 January 2021

Check for updates

\author{
Bryan A. Johnson ${ }^{1,14}$, Xuping Xie 2,14 , Adam L. Bailey,14, Birte Kalveram ${ }^{4}$, Kumari G. Lokugamage', \\ Antonio Muruato', Jing Zou' ${ }^{2}$, Xianwen Zhang ${ }^{2}$, Terry Juelich ${ }^{4}$, Jennifer K. Smith ${ }^{4}$, \\ Lihong Zhang ${ }^{4}$, Nathen Bopp ${ }^{4}$, Craig Schindewolf', Michelle Vu', Abigail Vanderheiden ${ }^{5,6}$, \\ Emma S. Winkler ${ }^{3,7}$, Daniele Swetnam ${ }^{2}$, Jessica A. Plante', Patricia Aguilar ${ }^{4}$, Kenneth S. Plante', \\ Vsevolod Popov ${ }^{4}$, Benhur Lee ${ }^{8}$, Scott C. Weaver ${ }^{1,9}$, Mehul S. Suthar ${ }^{5,6,10}$, Andrew L. Routh ${ }^{2}$, \\ Ping Ren ${ }^{4}$, Zhiqiang Ku" ${ }^{11}$, Zhiqiang An ${ }^{11}$, Kari Debbink ${ }^{12}$, Michael S. Diamond ${ }^{3,7,13}$, \\ Pei-Yong Shi ${ }^{2,9,15}$, Alexander N. Freiberg ${ }^{4,9,15}$ \& Vineet D. Menachery ${ }^{1,9,15} \bowtie$
}

\begin{abstract}
Severe acute respiratory syndrome coronavirus 2 (SARS-CoV-2)-a new coronavirus that has led to a worldwide pandemic ${ }^{1}$-has a furin cleavage site (PRRAR) in its spike protein that is absent in other group-2B coronaviruses ${ }^{2}$. To explore whether the furin cleavage site contributes to infection and pathogenesis in this virus, we generated a mutant SARS-CoV-2 that lacks the furin cleavage site ( $\triangle P R R A)$. Here we report that replicates of $\triangle$ PRRA SARS-CoV-2 had faster kinetics, improved fitness in Vero E6 cells and reduced spike protein processing, as compared to parental SARS-CoV-2. However, the $\triangle$ PRRA mutant had reduced replication in a human respiratory cell line and was attenuated in both hamster and K18-hACE2 transgenic mouse models of SARS-CoV-2 pathogenesis. Despite reduced disease, the $\triangle$ PRRA mutant conferred protection against rechallenge with the parental SARS-CoV-2. Importantly, the neutralization values of sera from patients with coronavirus disease 2019 (COVID-19) and monoclonal antibodies against the receptor-binding domain of SARS-CoV-2 were lower against the $\triangle$ PRRA mutant than against parental SARS-CoV-2, probably owing to an increased ratio of particles to plaque-forming units in infections with the former. Together, our results demonstrate a critical role for the furin cleavage site in infection with SARS-CoV-2 and highlight the importance of this site for evaluating the neutralization activities of antibodies.
\end{abstract}

The emergence of SARS-CoV-2 has led to the worldwide pandemic of COVID-19 ${ }^{1,3}$.SARS-CoV-2, similar to severe acute respiratory syndrome coronavirus (SARS-CoV) and Middle East respiratory syndrome coronavirus (MERS-CoV), induces severe respiratory disease that includes fever and multilobar pneumonia and-in many cases-leads to death ${ }^{4}$. Although SARS-CoV-2 has a similar genomic structure to and shares protein homology with SARS-CoV, the ability of the former to spread asymptomatically and cause mild-to-severe disease distinguishes the current pandemic from an earlier pandemic caused by SARS-CoV ${ }^{5}$. Studies that have examined the spike (S) protein-a glycoprotein that is responsible for receptor binding and entry, after cleavage at its $\mathrm{S} 1 / \mathrm{S} 2$ junction and S2 sites (Extended Data Fig. 1a) -indicate that SARS-CoV-2 has greater affinity for the ACE2 receptor than does SARS-CoV ${ }^{6}$. Notably, previous attention has been focused on a furin cleavage motif at the $\mathrm{S} 1 / \mathrm{S} 2$ cleavage site $^{2}$. Absent in other group-2B coronaviruses, four amino acids (PRRA) form a RXXR cleavage motif for serine proteases when added to S1/S2 cleavage site (PRRAR) ${ }^{7}$ (Extended Data Fig. 1b). Previous structural analysis has shown that furin cleavage facilitates the binding of a higher proportion of the S protein to the human ACE2 receptor ${ }^{8}$, and may have facilitated the emergence of SARS-CoV- 2 in humans. To date, the furin cleavage site has been analysed using mutated pseudotyped viruses that ablate the ability of the $S$ protein to mediate cell-cell and virus-cell fusion in Calu-3 cells (a human lung adenocarcinoma cell line $)^{9,10}$. Other studies have isolated deletion variants of SARS-CoV-2 that span the furin cleavage and S1/S2 site, which lead to attenuated infection $^{11,12}$. To our knowledge, no studies to date have evaluated the function of the furin cleavage site using authentic SARS-CoV-2 that contains a precise PRRA deletion. Such studies are necessary as discrepant results previously reported for the S(D614G) mutation highlight differences between pseudotyped and authentic SARS-CoV-2 variants ${ }^{13,14}$.

'Department of Microbiology and Immunology, University of Texas Medical Branch, Galveston, TX, USA. ²Department of Biochemistry and Molecular Biology, University of Texas Medical

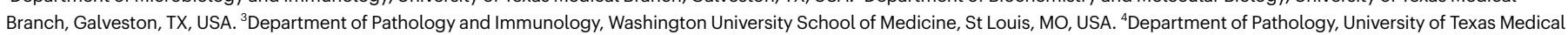

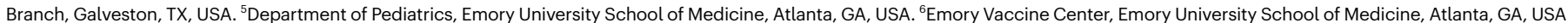
${ }^{7}$ Department of Medicine, Washington University School of Medicine, St Louis, MO, USA. ${ }^{8}$ Icahn School of Medicine at Mount Sinai, New York, NY, USA. ${ }^{9}$ Institute for Human Infection and Immunity, University of Texas Medical Branch, Galveston, TX, USA. ${ }^{10}$ Yerkes National Primate Research Center, Atlanta, GA, USA. ${ }^{11} T e x a s$ Therapeutics Institute, Brown Foundation Institute of

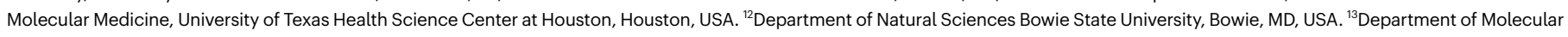
Microbiology, Washington University School of Medicine, St Louis, MO, USA. ${ }^{14}$ These authors contributed equally: Bryan A. Johnson, Xuping Xie, Adam L. Bailey. ${ }^{15}$ These authors jointly supervised this work: Pei Yong Shi, Alexander N. Freiberg, Vineet D. Menachery. ${ }^{\circledR e}$-mail: vimenach@utmb.edu 
Here we used a reverse genetic system to generate a SARS-CoV-2 mutant that lacks the furin cleavage site ( $\triangle$ PRRA) of the S protein ${ }^{15}$. The deletion of PRRA reduced $S$ protein cleavage, but augmented viral replication in Vero E6 cells. Ectopic expression of TMPRSS2 in Vero E6 cells removed the fitness advantage for $\triangle$ PRRA SARS-CoV-2. By contrast, the $\triangle$ PRRA mutant was attenuated in a human respiratory cell line and had reduced viral pathogenesis in both hamsters and K18-hACE2 transgenic mice (which express human ACE2). Notably, the $\triangle$ PRRA mutation required more antisera and monoclonal antibodies targeting the receptor-binding domain (RBD) of the S protein for neutralization. Our results demonstrate a critical role of the furin cleavage site in SARS-CoV-2 infection, and highlight concerns with using deletion variants in which this site is absent for research into COVID-19.

\section{Generation of the $\triangle P R R A$ mutant}

We generated mutant virus that lacks the PRRA motif using a SARS-CoV-2 reverse genetic system ${ }^{15}$ (Fig. 1a). The furin cleavage site resides in an exterior, and ostensibly unresolved, loop of the structure of the S protein of SARS-CoV-2, below the globular head and away from the RBD (Fig. 1b). We used homology modelling to visualize the PRRA site in this extended loop (shown in cyan in Fig. 1b). Deletion of the PRRA motif is predicted to shorten the loop without disrupting the overall structure of the $S$ protein. Following electroporation, we recovered $\triangle$ PRRA SARS-CoV-2 with a titre equivalent to the wild-type virus. $\triangle$ PRRA SARS-CoV-2 produced a larger plaque size on Vero E6 cells than did wild-type virus, which suggests that there are changes in viral replication and spread in the absence of the furin cleavage site (Extended Data Fig. 1c).

\section{Distinct $\triangle P R R A$ replication and cleavage}

To evaluate viral replication, we inoculated Vero E6 cells with wild-type and $\triangle$ PRRA SARS-CoV-2.SARS-CoV-2 replicates robustly in Vero E6 cells, which often are used for the propagation of virus stock and production of inactivated vaccine ${ }^{16}$. Following inoculation at a low multiplicity of infection (MOI) of 0.01 plaque-forming units (PFU) per cell, both wild-type and $\triangle$ PRRASARS-CoV-2 replicated to similar endpoint titres. However, $\triangle$ PRRA mutant had a 25 -fold-higher viral titre and greater cytopathic effect at $24 \mathrm{~h}$ after infection (Fig. 1c). Thus, the loss of the furin cleavage site augments SARS-CoV-2 replication in Vero E6 cells.

We next evaluated $S$ processing of $\triangle$ PRRA SARS-CoV-2 relative to wild-type SARS-CoV-2 and SARS-CoV. We inoculated Vero E6 cells at an MOI of about 0.1 for $24 \mathrm{~h}$, and isolated purified virions using sucrose cushion ultracentrifugation. We then examined the pelleted for S processing and nucleocapsid $(\mathrm{N})$ protein by western blotting. After infection with SARS-CoV, the majority of the $S$ protein is present in its full-length form (98.6\%) (Fig. 1d, Extended Data Fig. 2a; uncropped images are provided in Supplementary Figs. 1,2), consistent with minimal processing. By contrast, virions of wild-type SARS-CoV-2 had substantial S protein cleavage; $59.6 \%$ of the S protein was cleaved to S1/S2 products. The $\triangle$ PRRA mutant reduced the amount of S1/S2 cleavage to $14.5 \%$. Given the similar levels of $\mathrm{N}$ protein in all cases, these results illustrate differences between SARS-CoV and SARS-CoV-2, and show that SARS-CoV-2 processing of the S protein is driven by the furin cleavage site.

Given the replication advantage at $24 \mathrm{~h}$ after infection (Fig. 1d), we evaluated the fitness of $\triangle$ PRRA SARS-CoV-2 relative to wild-type SARS-CoV-2 in a competition assay. Using PFU to determine the input, we mixed the wild type and mutant at different ratios in Vero E6 cells, and used quantitative PCR with reverse transcription (RT-qPCR) to quantify relative fitness at $24 \mathrm{~h}$ after infection (Fig. 1e, Extended Data Fig. 2b, c). At a 50:50 ratio, the $\triangle$ PRRA mutant outcompeted wild-type virus, and had become nearly $90 \%$ of the viral population at $24 \mathrm{~h}$. A 90:10 input ratio of wild type:mutant resulted in the $\triangle$ PRRA mutant comprising around $65 \%$ of the viral sequences at $24 \mathrm{~h}$. The inverse input ratio of 10:90 wild type:mutant produced 97\% $\triangle$ PRRA SARS-CoV-2, which further confirmed the advantage of the mutant in Vero E6 cells. We corroborated the RT-qPCR results with deep sequencing analysis (Extended Data Fig. 2d). Thus, deletion of the furin cleavage site provides a fitness advantage in Vero E6 cells, and may contribute to mutations found in wild-type SARS-CoV-2 cultured on Vero E6 cells.

\section{Attenuation of $\triangle$ PRRA in Calu-3 cells}

We next evaluated the $\triangle$ PRRA mutant in Calu-3 2B4 cells, which are commonly used to study other coronaviruses and influenza virus ${ }^{17}$. In contrast to results in Vero E6 cells, $\triangle$ PRRA SARS-CoV-2 replicated less efficiently in Calu-3 cells than did wild-type SARS-CoV-2 (Fig. 1f). The $\triangle$ PRRA mutant had about 10 -fold reductions in viral titre at both 48 and $72 \mathrm{~h}$ after infection, which indicates that the loss of the furin cleavage site impairs viral replication in Calu- 3 cells. Next, we evaluated $\mathrm{S}$ processing on virions that were produced from Calu-32B4 cells. Consistent with results in Vero E6 cells, S protein from SARS-CoV was not cleaved to the S1/S2 form. However, about $87.3 \%$ of the S protein from wild-type SARS-CoV-2 was processed to the S1/S2 form-a greater amount than that observed in Vero E6 cells (59.6\%) (Fig. 1g, Extended Data Fig. 2e; uncropped images are provided in Supplementary Figs.1, 2). The $\triangle$ PRRA mutant also showed an increase in the S1/S2 cleavage product. This band represented more than twice as much $\mathrm{S} 1 / \mathrm{S} 2$ cleavage product (33.1\% versus $14.5 \%)$ than that seen in Vero E6 cell supernatants, although the deletion did result in a major shift from cleaved to uncleaved S protein. Although more full-length S protein is observed in infection with the wild-type virus, the results in Calu-3 cells indicate that-even without the furin cleavage site-there is substantial processing of the SARS-CoV-2S protein. Thus, factors outside of the PRRA motif contribute to cleavage of the SARS-CoV-2 S protein in a cell-type-dependent manner.

\section{TMPRSS2 reduces fitness advantage of $\triangle P R R A$}

One distinction between Vero E6 and Calu-3 cells is the expression of host serine proteases, such as TMPRSS $2^{18}$. To determine whether this cell-surface protease modulates replication of $\triangle$ PRRA SARS-CoV-2, we evaluated infection in Vero E6 cells that ectopically express TMPRSS2. Following infection at a low MOI (0.01), wild-type and $\triangle$ PRRA SARS-CoV-2 replicated to similar levels (Fig. 1h). Next, we performed a competition assay to evaluate the fitness of the $\triangle$ PRRA mutant in the TMPRSS2-expressing Vero E6 cells. At a 50:50 input ratio, the $\triangle$ PRRA mutant and wild-type virus remained at equal levels in TMPRSS2-expressing Vero E6 cells (Fig. 1i). These results demonstrate that the expression of TMPRSS2 reduced the replication and fitness advantage of $\triangle$ PRRA SARS-CoV-2 over wild-type SARS-CoV-2 in Vero E6 cells. To examine whether TMPRSS 2 affects $S$ cleavage, we purified virions from TMPRSS2-expressing Vero E6 cells and quantified the ratio of full-length S protein to the S1/S2 form (Fig. 1j; uncropped images are provided in Supplementary Figs. 3, 4). Compared with results in Vero E6 cells, the expression of TMPRSS2 had minimal effect on S processing ratios (Extended Data Fig. 2a, f). In both SARS-CoV and $\triangle$ PRRA SARS-CoV-2, the full-length form of S protein was mostly intact $(96.4 \%$ and $95.9 \%$, respectively), whereas wild-type SARS-CoV-2 had nearly half of the S protein processed to the S1/S2 form (Extended Data Fig. 2f). Overall, the results indicate that (i) TMPRSS2 affects virus entry rather than virion release and (ii) TMPRSS2-expressing Vero E6 cells reduce selection of $\triangle$ PRRA mutants.

\section{In vivo attenuation of $\triangle P R R A$ mutant}

We next evaluated the $\triangle$ PRRA mutant in vivo using a hamster model of SARS-CoV-2 pathogenesis ${ }^{19}$, using four male hamsters challenged 
a

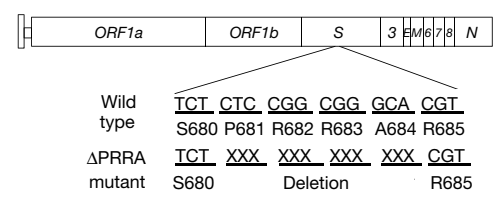

type $\quad$ S680 P681 R682 R683 $\frac{\mathrm{A} 684}{\mathrm{R} 685}$

mutant $\frac{\mathrm{TCT}}{\mathrm{S} 680} \frac{\mathrm{XXX}}{\text { Deletion }} \frac{\mathrm{XXX}}{\mathrm{XXX}} \frac{\mathrm{XXX}}{\mathrm{R} 685}$

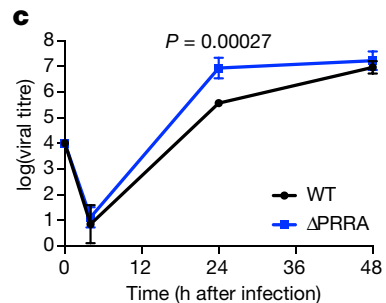

d

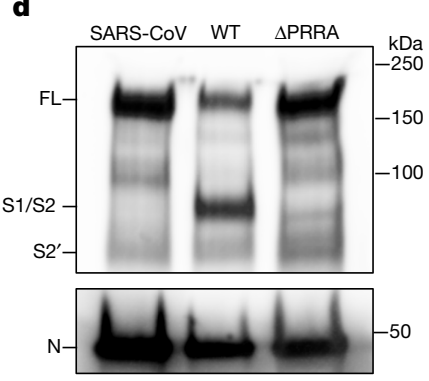

h

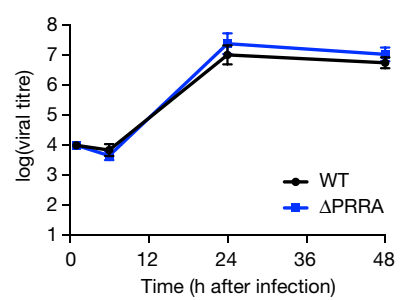

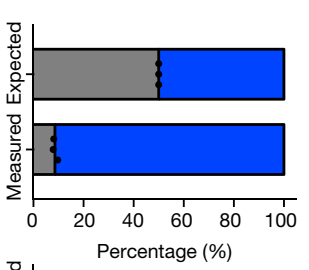

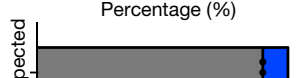

b

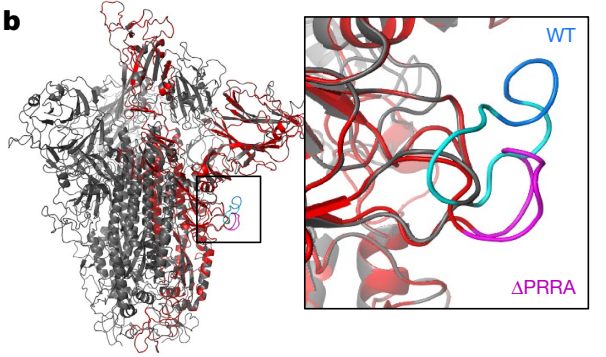

f
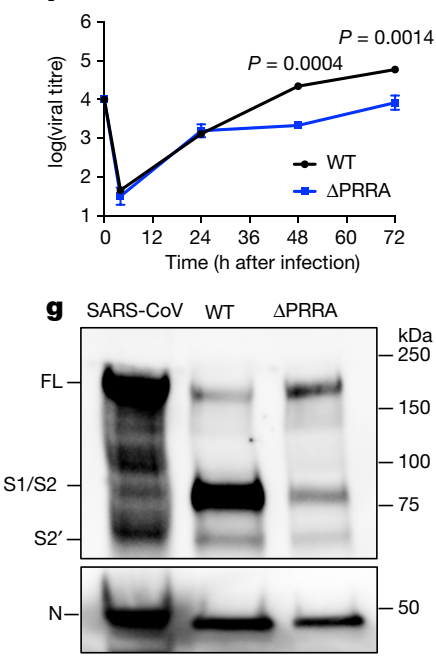

\footnotetext{
(1)
}

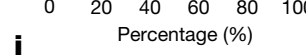

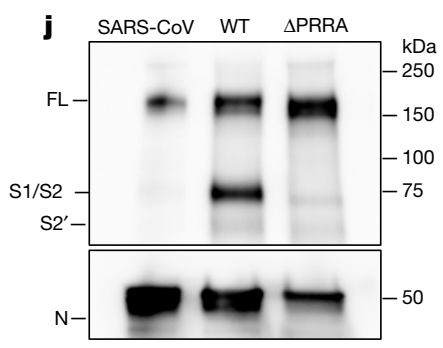

Fig. 1 | Distinct replication, $S$ cleavage and competition for $\triangle P R R A$ SARS-CoV-2. a, Schematic of SARS-CoV-2, showing deletion of the furin cleavage site. $O R F 3, O R F 6, O R F 7$ and $O R F 8$ are designated $3,6,7$ and 8 , respectively.b,SARS-CoV-2 trimer (grey) with $\triangle$ PRRA mutant monomer overlaid (red). The loop (inset) shows wild-type SARS-CoV-2 (WT) (cyan) with the PRRA sequence (blue) and $\triangle$ PRRA mutant (pink). Models were generated using the structure of SARS- $\mathrm{CoV}$ (Protein Data Bank code 6ACD). c, Viral titre from Vero E6 cells infected with wild-type (black) or $\triangle$ PRRA (blue) SARS-CoV-2 at an MOI of $0.01(n=3)$. d, Purified SARS-CoV, wild-type SARS-CoV-2 and $\triangle$ PRRA SARS-CoV-2 virions from Vero E6 cells probed with anti-S (top) or anti-N antibody (bottom). Full length (FL), S1/S2 cleavage form and S2' are annotated. Results are representative of two independent experiments. e, Competition assay between wild-type (black) and $\triangle$ PRRA (blue) SARS-CoV-2, showing RNA percentage based on RT-qPCR at 50:50 (top), 90:10 (middle) and 10:90 (bottom) ratios of wild type: $\triangle \mathrm{PRRA}(n=3$ per group).f, Viral titre from Calu- 3 2B4 cells infected with wild-type (black) or $\triangle$ PRRA (blue) SARS-CoV-2 at an MOI of $0.01(n=3)$. g, Purified SARS-CoV, wild-type SARS-CoV-2 and $\triangle$ PRRA SARS-CoV-2 virions from Calu-32B4 cells probed with anti-S (top) or anti-N (bottom) antibody. Results are representative of two independent experiments.h, Viral titre from Vero E6 cells expressing TMPRSS2, infected with wild-type (black) or $\triangle$ PRRA (blue) SARS-CoV-2 at an MOI of $0.01(n=5)$. i, Competition assay between wild-type (black) and $\triangle$ PRRA (blue) SARS-CoV-2 in Vero E6 cells expressing TMPRSS2, showing RNA percentage based on RT-qPCR at 50:50 ratio of wild type: $\triangle$ PRRA ( $n=3$ per group).j, Purified SARS-CoV, wild-type SARS-CoV-2 and $\triangle$ PRRA SARS-CoV-2 virions from Vero E6 cells expressing TMPRSS2, probed with anti-S (top) or anti-N (bottom) antibody. Results are representative of two independent experiments. Data are mean \pm s.d. in c, e, $\mathbf{f}, \mathbf{h}, \mathbf{i}$. $P$ values from two-tailed Student's $t$-test. via intranasal inoculation with $10^{5} \mathrm{PFU}$ of wild-type SARS-CoV-2 or the $\triangle$ PRRA mutant (Fig. 2a). After infection with wild-type SARS-CoV-2, hamsters steadily lost weight starting at day 2 and continuing through to day 8 , with peak weight loss of almost $15 \%$ (Fig. 2b, Extended Data Fig. 3a). Disease scores peaked at day 8 , at which point hamsters had ruffled fur, hunched posture and reduced activity that required additional monitoring (Fig. 2c, Extended Data Fig. 3b). Despite severe disease, the hamsters infected with wild-type SARS-CoV-2 recovered and regained their starting weight by day 15 (Extended Data Fig. 3a). By contrast, hamsters infected with $\triangle$ PRRA SARS-CoV-2 showed minimal weight loss and no disease (Fig. 2b, Extended Data Fig. 3a, b). In both infection groups, hamsters gained weight after day 10 over the remainder of the 28-day time course (Extended Data Fig. 3a). In nasal washes, hamsters infected with wild-type or $\triangle$ PRRA SARS-CoV-2 had similar viral titres at two days after infection (Fig. 2d). However, greater replication of $\triangle P R R A$ SARS-CoV-2 was observed at three and four days after infection than was seen with wild-type SARS-CoV-2. In addition, the wild-type virus was cleared from the nasal washes a day earlier than was the $\triangle$ PRRA mutant, although no infectious virus was detected after day 7 in either hamster group. For viral RNA from oral swabs, we observed a similar pattern, in which higher viral RNA concentrations were seen at three and four days after infection with the $\triangle$ PRRA mutant relative to wild-type virus 

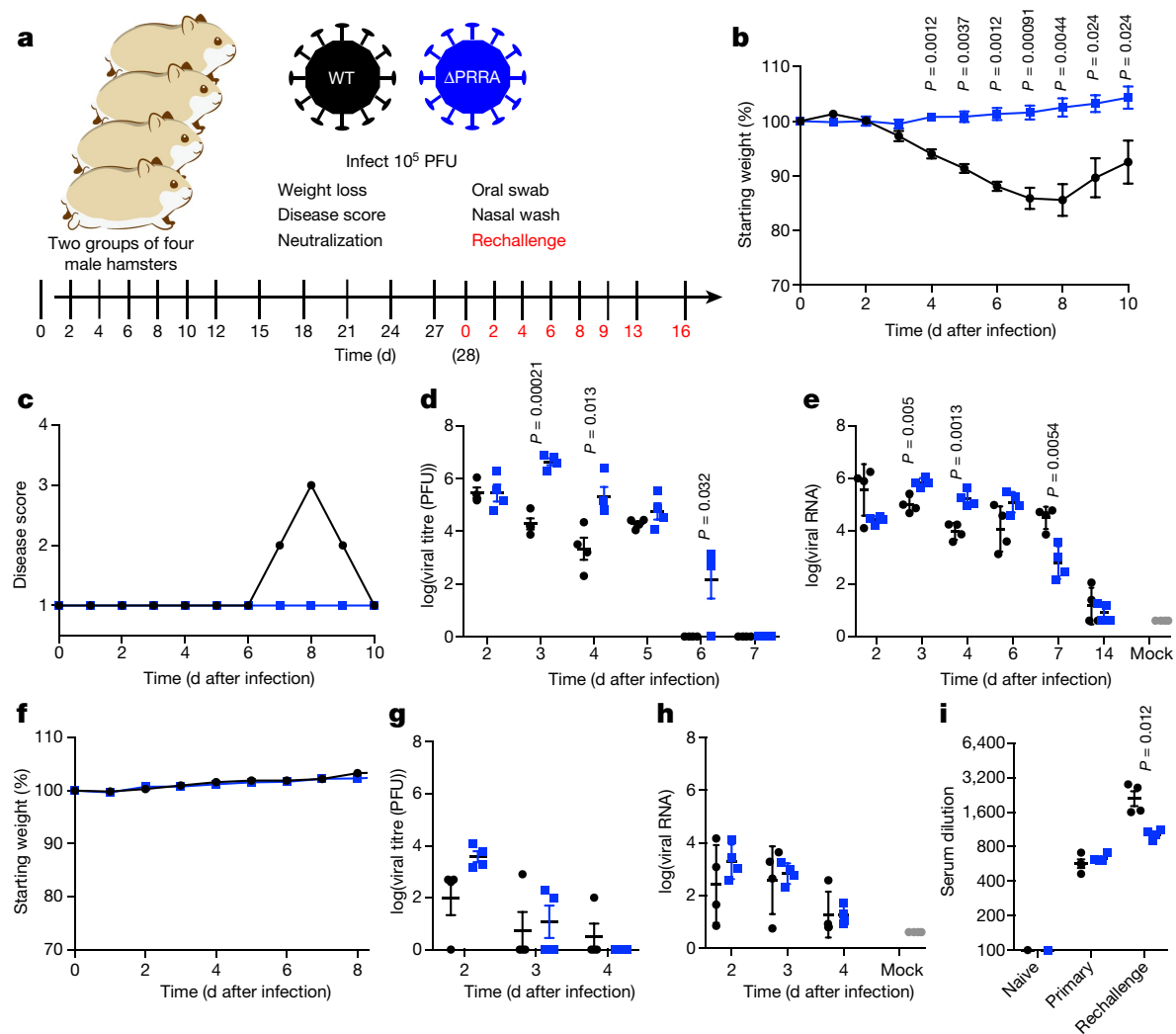

Fig. 2 Hamster infections with $\triangle$ PRRA SARS-CoV-2. a, Schematic for primary challenge with SARS-CoV-2. b-e, Two groups of male hamsters ( $n=4$ in each group) were challenged with $10^{5} \mathrm{PFU}$ of wild-type (black) or $\triangle \mathrm{PRRA}$ (blue) SARS-CoV-2, and evaluated for weight loss (b), disease score (c), viral titre from nasal wash (d) and viral RNA from oral swabs (e).f-i, Twenty-eight days after infection, hamsters infected with wild-type (black) or $\triangle$ PRRA (blue)
SARS-CoV-2 were rechallenged with $10^{5}$ PFU of wild-type SARS-CoV-2 and evaluated for weight loss (f), viral titre from nasal wash (g), viral RNA from oral swabs (h) and PRNT $_{50}$ dilution using sera from hamsters after primary challenge and rechallenge (i). Data are mean \pm s.e.m. $P$ values from two-tailed Student's $t$-test.
(Fig. 2e). However, the viral RNA in the swabs stayed positive though to seven days after infection, with higher concentrations been found in hamsters infected with wild-type virus than those infected with $\triangle$ PRRA SARS-CoV-2. Together, these results suggest that-despite attenuated disease-the $\triangle$ PRRA mutant replicates efficiently in the oral and nasal cavity of hamsters

\section{$\triangle P R R A$ mutant protects from SARS-CoV-2 rechallenge}

Because some vaccine strategies mutate the furin cleavage $\operatorname{site}^{20,21}$, we evaluated whether infection with $\triangle$ PRRA SARS-CoV-2 protects from rechallenge with wild-type SARS-CoV-2. We rechallenged hamsters that had previously been infected with wild-type or $\triangle$ PRRASARS-CoV-2 with $10^{5}$ PFU of wild-type SARS-CoV-2 at 28 days after the primary challenge. Hamsters initially infected with either wild-type or $\triangle$ PRRA SARS-CoV-2 were both protected from weight loss after rechallenge (Fig. 2f, Extended Data Fig. 3b). However, mild disease (ruffled fur) was observed in one hamster that had previously been infected with wild-type SARS-CoV-2 (Extended Data Fig. 3c). By contrast, hamsters that had been infected with the $\triangle$ PRRA exhibited neither weight loss nor disease. Nasal wash titres and viral RNA from oral swabs were significantly reduced compared to the initial infection in both groups, and infectious virus was cleared by four days after infection (Fig. $2 \mathrm{~g}, \mathrm{~h}$ ). Primary infection with both $\triangle$ PRRA and wild-type SARS-CoV-2 produced neutralizing antibodies in serum (about $1 / 600$ each at day 28) (Fig. 2i) and subsequent rechallenge boosted this activity, although we noted a twofold difference in the final half-maximal plaque-reduction neutralizing titre $\left(\mathrm{PRNT}_{50}\right)$ with wild-type versus $\triangle$ PRRA SARS-CoV-2 (about $1 / 2,000$ and about $1 / 1,000$, respectively). Together, these results indicate the attenuated infection with the $\triangle$ PRRA mutant induces sufficient immunity to protect hamsters from rechallenge with wild-type SARS-CoV-2.

\section{$\triangle P R R A$ lung disease is attenuated in K18-hACE2 mice}

To further evaluate pathogenesis, we infected transgenic C57BL/6 mice that express human ACE2 and are permissive for SARS-CoV-2 infection $^{22}$. We inoculated male and female K18-hACE2 mice via the intranasal route with $10^{3}$ PFU of wild-type or $\triangle$ PRRA SARS-CoV-2 (Fig. 3a). K18-hACE2 mice infected with wild-type SARS-CoV-2 lost significantly more weight than those infected with $\triangle$ PRRA, starting at four days after infection and continuing through to the end of the experiment (Fig. 3b). Attenuated disease corresponded to reduced viral replication at day 2 in the lung, nasal turbinates and nasal washes (Fig. 3c-e). However, significant differences in viral burden were not observed in lungs or brain at seven days after infection (Fig. 3f). To examine changes to the functional properties of the lung, we mechanically ventilated mice via tracheostomy and measured several respiratory biophysical parameters. As compared to infection with $\triangle$ PRRA SARS-CoV-2, K18-hACE2 mice infected with wild-type SARS-CoV-2 had reduced inspiratory capacity (Fig. 3g), a downward deflection in the pressure-volume loop (Fig. $3 \mathrm{~h}$ ) and increased tissue dampening, respiratory resistance and tissue elastance, consistent with restrictive lung disease localized to the alveoli and tissue parenchyma (Extended Data Fig. 4a-d). By contrast, only mild changes in pulmonary mechanics were observed in mice infected with the $\triangle$ PRRA 
a

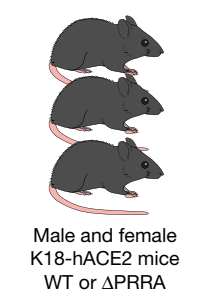

d

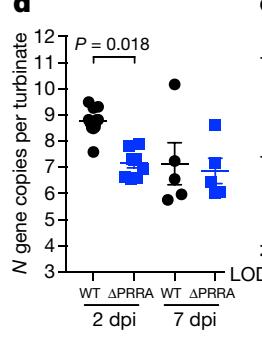

i

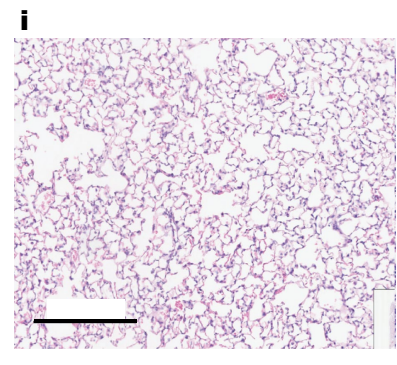

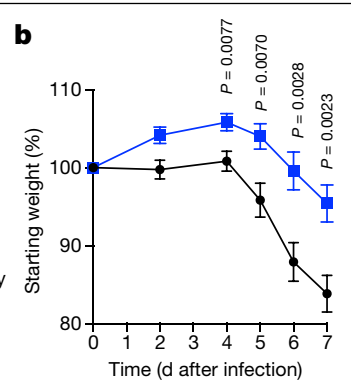

f

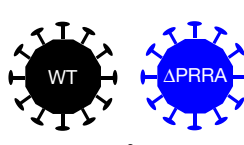

Infect $10^{3} \mathrm{PFU}$

Weight loss Nasal swab

Lung

ung function Histopathology

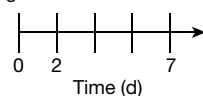

e

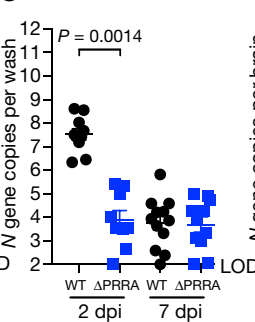

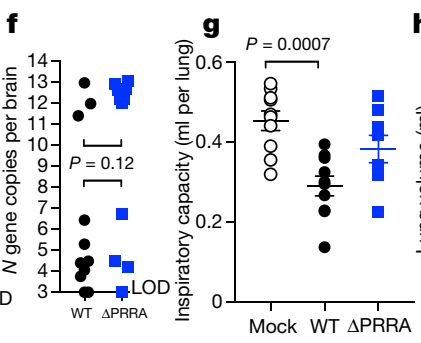

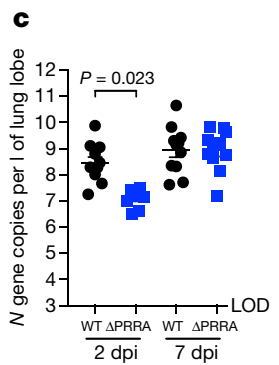

h

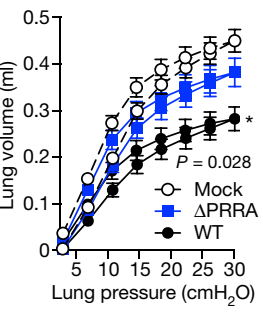

j $\quad k$

$\mathbf{k}$

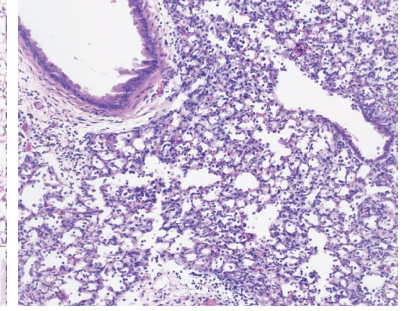

ter
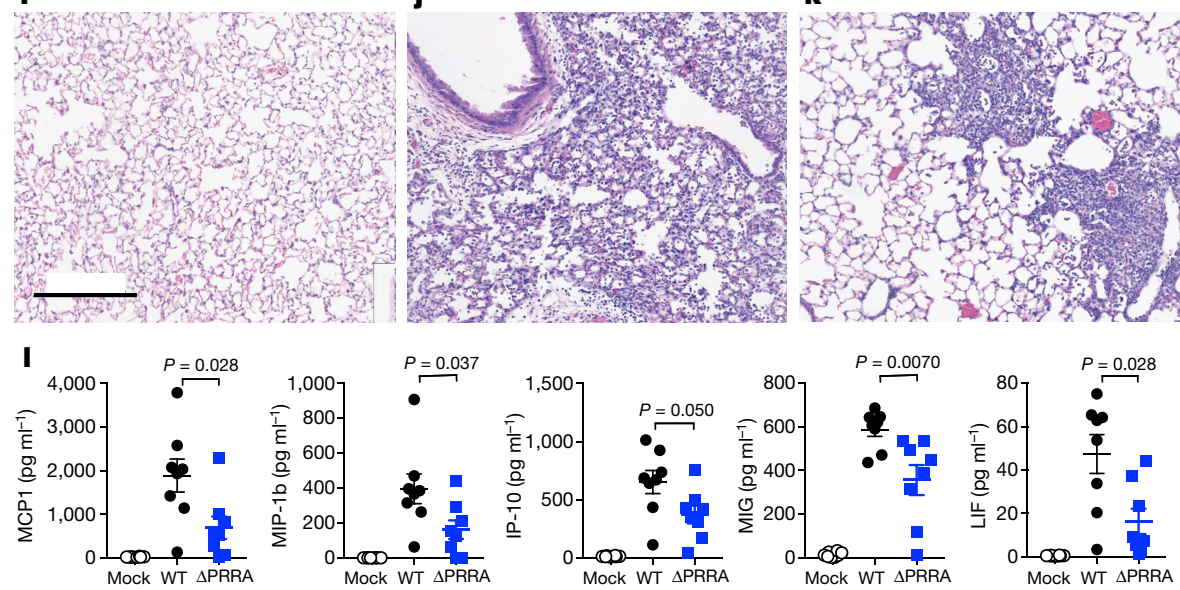

Fig. 3 | Infection of K18-hACE2 transgenic mice with $\triangle$ PRRA SARS-CoV-2. a, Schematic of SARS-CoV-2 challenge, created with BioRender. b-f, Male and female mice were challenged with $10^{3} \mathrm{PFU}$ of wild-type (black) or $\triangle$ PRRA (blue) SARS-CoV-2, and evaluated for weight loss ( $n=12$ for both groups) (b), and viral RNA from the lung (c), nasal turbinate (d), nasal wash (e) and brain (f). At 2 days post-infection (dpi), $n=9$ mice infected with wild type, 11 mice infected with $\triangle$ PRRA; at $7 \mathrm{dpi}, n=11$ mice for both. $N$ gene copies in the brain were measured at $7 \mathrm{dpi}$ only. LOD, limit of detection. $\mathbf{g}$, $\mathbf{h}$, Lung function evaluated at $7 \mathrm{dpi}$ using Flexivent mechanical ventilator to assess inspiratory capacity $(\mathbf{g})$ and pressure-volume loop (h). $n=10$ mice infected with wild type; $n=9$ mice infected with $\triangle$ PRRA. $\mathbf{i}-\mathbf{k}$, Lung histopathology at $7 \mathrm{dpi}$ from mock- (i), wild-type- $(\mathbf{j})$ and $\triangle$ PRRA- (j) infected mice. Images are representative of lung sections from three mice in all cases. Scale bar, $250 \mu \mathrm{m}$. I, Chemokine analysis of mouse lung homogenates at $7 \mathrm{dpi}$, from mock-infected mice (white), or mice infected with wild-type (black) or $\triangle$ PRRA (blue) SARS-CoV-2. $n=8$ for all groups. Data are mean \pm s.e.m. $P$ values from two-tailed Student's $t$-test with unequal variance (b), Kruskal-Wallis Test for multiple comparisons (c-e, $\mathbf{g}, \mathbf{h}), \chi^{2}$ test (f) or a two-tailed Mann-Whitney test between wild-type and $\triangle$ PRRA (I).

\section{Assessing antibody neutralization titres for $\triangle P R R A$}

We next evaluated the effect of deletion of the furin cleavage site on virus neutralization. To quantify neutralization, we generated a $\triangle$ PRRA mutant that contains a $m$ NeonGreen reporter in open reading frame 7 (ORF7) and compared results to the wild-type SARS-CoV-2 $\left(\right.$ ref. $^{23}$ ) (Fig. 4a). Examining sera from 17 individuals with COVID-19, we found a nearly uniform reduction in $\mathrm{PRNT}_{50}$ values against the $\triangle \mathrm{PRRA}$ mutant versus wild-type viurs (Fig. 4b). The lower PRNT $_{50}$ values were observed in COVID-19 serum samples with low, intermediate and high neutralizing activity (Fig. 4c-e), and averaged a 2.3-fold reduction across the 17 sera tested. The differences between wild-type and $\triangle$ PRRA SARS-CoV-2 were significant for samples with high and intermediate neutralizing activity, but below threshold for the samples with low neutralizing activity (owing to incomplete neutralization) (Supplementary Table 1). The consistency in the reduction may be due to: (1) the fact that the S proteins have an altered conformation in the $\triangle$ PRRA virions, 

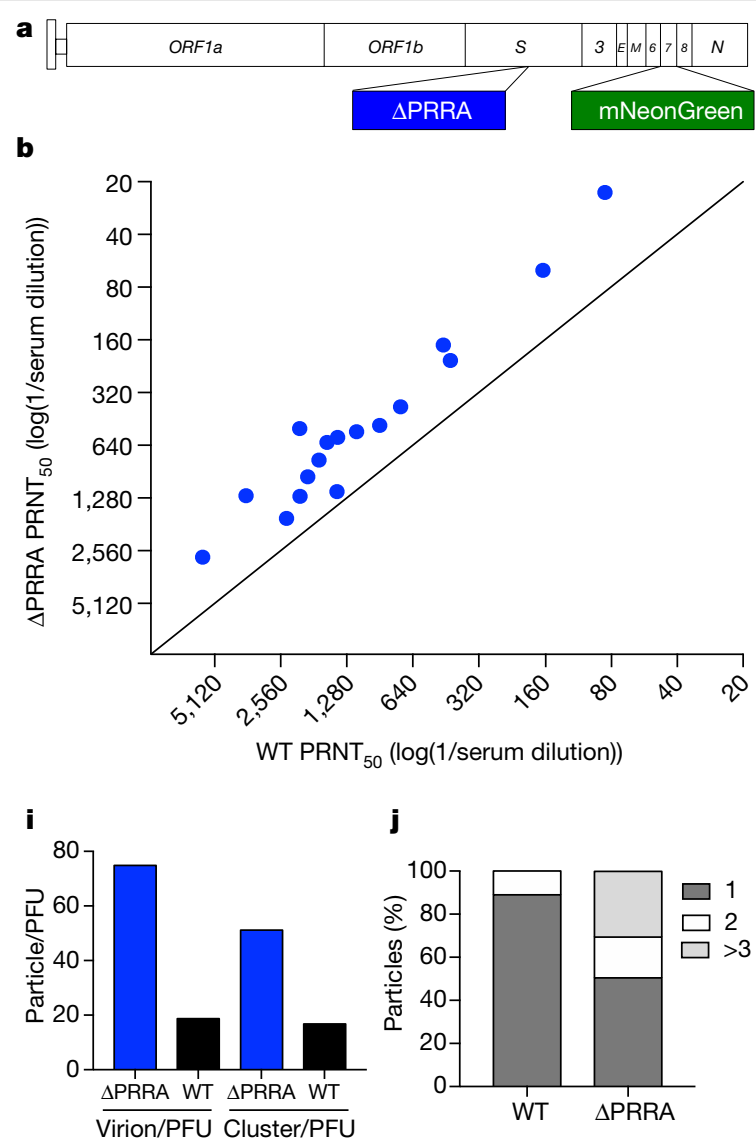

Fig. 4 | Assessing antibody neutralization of $\triangle$ PRRA SARS-CoV-2. a, Schematic for $\triangle$ PRRA SARS-CoV-2 reporter virus expressing $\mathrm{mNe}$ NonGreen gene in place of $O R F 7 . \mathbf{b}, \mathrm{PRNT}_{50}$ values measured by mNeonGreen expression, with wild-type SARS-CoV-2 on the $x$ axis and $\triangle$ PRRA SARS-CoV-2 on the $y$ axis. c-e, Representative curves from low (c), intermediate (d) and high (e)
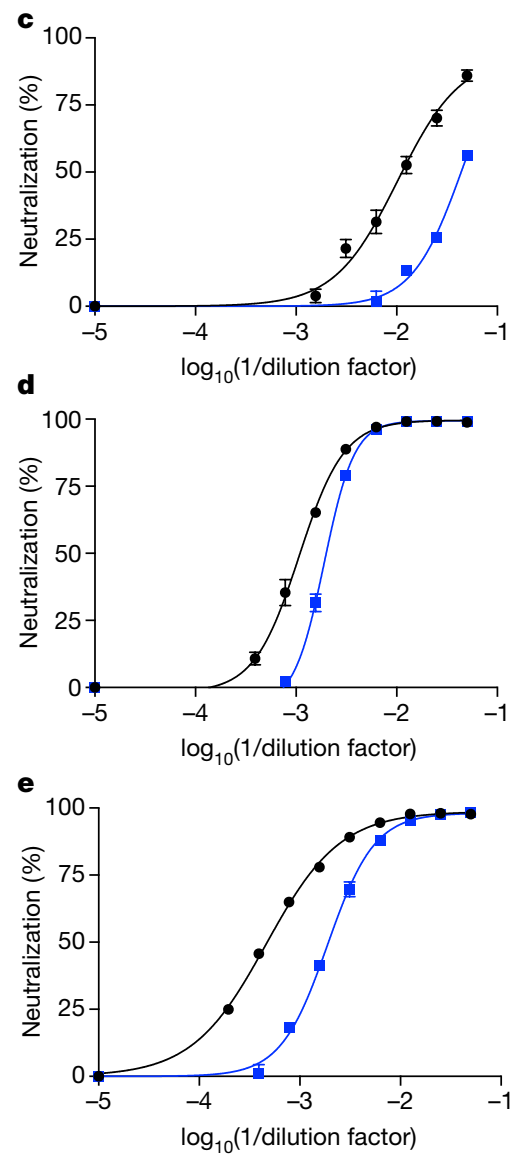

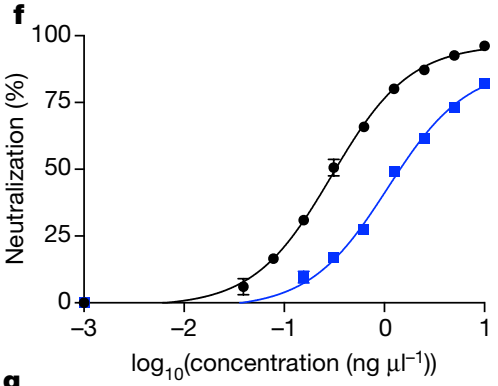

$\mathbf{g}$

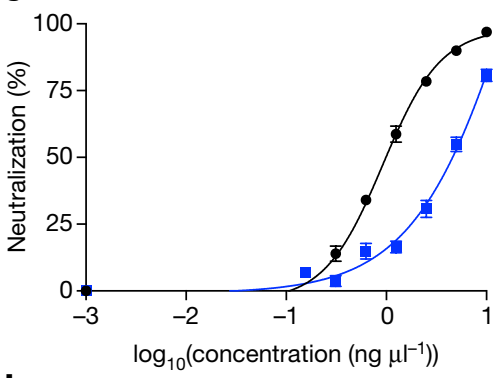

h

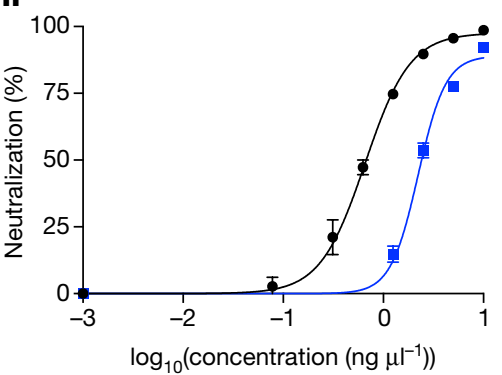

neutralizing sera from patients with COVID-19. $n=3 . \mathbf{f}-\mathbf{h}$, Neutralization curves from three monoclonal antibodies (mAb1 (f), mAb 2 (g) and mAb3 (h)). $n=3$. i, Particle:PFU ratio determined from 40 fields, dividing into individual particles (left) and clusters (right) to determine ratios.j, Percentage of particles as individual virions (1), doubles (2) or larger clusters ( $>3)$. Data are mean \pm s.e.m.

restricting access to more-cryptic sites on the S protein and resulting in wild-type virus being more readily neutralized by non-RBD antibodies; and (2) the presence of more-intact $S$ molecules on the virion surface of $\triangle$ PRRA virions, requiring more antibodies to neutralize $\triangle$ PRRA than wild-type SARS-CoV-2. To explore these possibilities, we evaluated neutralization of the $\triangle$ PRRA mutant by three monoclonal antibodies that target the SARS-CoV-2 RBD (Fig. $4 \mathrm{f}-\mathrm{h}$ ). Each monoclonal antibody targets a different site in the RBD, but they all showed similar reductions in neutralization of wild-type and $\triangle$ PRRA SARS-CoV-2. Whereas the results with monoclonal antibodies 1 and 3 reached significance, low neutralization levels precluded such conclusions with monoclonal antibody 2 (Supplementary Table 1). Together, these results highlight differences in antibody neutralization profiles between wild-type and $\triangle$ PRRA SARS-CoV-2.

Differences in the $\mathrm{PRNT}_{50}$ values suggest potential physical variation between wild-type and $\triangle$ PRRA virions. One possible explanation is that the $\triangle$ PRRA mutant has more full-length $\mathrm{S}$ protein than does wild-type SARS-CoV-2 (Fig. 1d, Extended Data Fig. 2a), which requires more antibody for neutralization. To explore this idea, we examined the wild-type and $\triangle$ PRRA virion by transmission electron microscopy. We evaluated 40 transmission electron microscopy fields of virions from wild-type and $\triangle$ PRRA stocks for morphology and to determine the particle to PFU ratio. Consistent with previous reports ${ }^{24}$, wild-type SARS-CoV-2 had classic morphology, formed single virions and had a particle:PFU ratio that approached 20 (Fig. 4i, Extended Data Fig. 5). By contrast, the $\triangle$ PRRA stocks showed a particle:PFU ratio that approached 80 , and nearly $33 \%$ of the particles clustered in groups of more than 3 virions (Fig. $4 \mathrm{j}$ ). The results suggest that the $\triangle$ PRRA mutant forms clusters of viruses, reminiscent of the cloaked virions of norovirus and hepatitis $\mathrm{C}$ virus ${ }^{25,26}$-although the mechanism for SARS-CoV-2 clumping remains unclear. When controlling for clumps as a single PFU, the particle:PFU ratio in $\triangle$ PRRA stocks drops to approximately 60:1 and is more consistent with the two- to threefold increase in serum required for neutralization of the $\triangle$ PRRA mutant. Combined with the increased expression of full-length $S$ protein, the clumping results and particle:PFU ratio provide several explanations for why the antibody concentrations required for neutralization are higher with $\triangle$ PRRA SARS-CoV-2.

Overall, the loss of the furin cleavage site in the SARS-CoV-2S protein has a major effect on infection and pathogenesis, with reduced replication in Calu-3 respiratory cells and ablated disease in two animal models of SARS-CoV-2 pathogenesis. These results are consistent with previous studies that have examined in furin cleavage site ${ }^{11,12}$. However, despite attenuated disease, the $\triangle$ PRRA mutant has some replication advantages over wild-type SARS-CoV-2 that may lead to cell-culture adaptions, which might complicate results. The fitness advantage of the $\triangle$ PRRA mutant in Vero E6 cells is consistent with previous reports of deletions of the furin cleavage site in SARS-CoV-2 preparations and samples from patients with COVID-19 ${ }^{11,12}$. This has implications for manufacturing inactivated COVID-19 vaccine on Vero cells ${ }^{27}$, and the shift in antibody neutralization of the $\triangle$ PRRA virus indicates the possibility of imprecise results if the furin cleavage site is affected ${ }^{28}$. As 
decisions regarding vaccines and therapeutic agents potentially rely on neutralization values, accuracy is an important issue.

A number of approaches can prevent the emergence of the $\triangle$ PRRA mutation in SARS-CoV-2 stocks. In our studies, we found no evidence for PRRA deletion in infectious clone-derived wild-type SARS-CoV-2 through passage 2. By using low-passage stocks, the incorporation of this mutation was limited. One alternative is the use of Vero E6 cells expressing TMPRSS2, which removes the fitness advantage of the $\triangle$ PRRA mutant and will allow virus propagation without altering the full-length-to-S1/S2 processing ratio of the S protein. However, continued passage risks this or other tissue culture adaptations, and careful monitoring of stock composition is needed. Using plaque purification techniques, wild-type SARS-CoV-2 can be selected for by its smaller plaque morphology.

The furin cleavage site promotes increased S cleavage in SARS-CoV-2, which has implications for pathogenesis. As such, vaccines and therapeutic agents that target the furin cleavage site offer an attractive approach to disrupt COVID- $19^{20}$ and could have implications for other coronaviruses with furin cleavage sites (including HKU1-CoV, OC43-CoV and MERS-CoV). Disruption or improvement of these furin sites could change the trajectory of diseases caused by these coronaviruses. In our studies, the $\triangle$ PRRA mutant attenuates disease in vivo and confers protection from subsequent rechallenge. However, $\triangle \mathrm{PRRA}$ replication was not ablated, and substantial tissue damage was observed in both of the in vivo pathogenesis models. Importantly, differences in the $\mathrm{PRNT}_{50}$ values suggest potential antigenic differences between wild-type and $\triangle$ PRRASARS-CoV-2 that could affect vaccination approaches. Strategies that disrupt or ablate the furin cleavage site might result in altered adaptive immune responses if the mutations occur in dominant epitopes. For example, on the basis of structural studies, loss of the furin cleavage site could reduce access to the more-open form of the S protein necessary to interact with human ACE2 receptor and alter the targets for antibody generation ${ }^{8}$. Although the attenuation of the $\triangle$ PRRA mutant holds promise for developing vaccines, further studies are needed to fully explore the extent of antibody and cell-based immunity induced by this mutant.

Overall, our data illustrate the critical role of the furin cleavage site in SARS-CoV-2 infection and pathogenesis. In its absence, the $\triangle$ PRRA mutant is attenuated in its ability to replicate in some cell types and to cause disease in vivo. However, the results are complicated by augmented replication and fitness in Vero E6 cells, which is driven by the absence of TMPRSS2 expression. Similarly, altered antibody neutralization profiles indicate a critical need to survey this mutation in the analysis of SARS-CoV-2 treatments and vaccines. Our work highlights the critical nature of the furin cleavage site in understanding $S$ protein biology and SARS-CoV-2 infection and pathogenesis.

\section{Online content}

Any methods, additional references, Nature Research reporting summaries, source data, extended data, supplementary information, acknowledgements, peer review information; details of author contributions and competing interests; and statements of data and code availability are available at https://doi.org/10.1038/s41586-021-03237-4.

1. Gralinski, L. E. \& Menachery, V. D. Return of the coronavirus: 2019-nCoV. Viruses 12, 135 (2020).

2. Coutard, B. et al. The spike glycoprotein of the new coronavirus 2019-nCoV contains a furin-like cleavage site absent in CoV of the same clade. Antiviral Res. 176, 104742 (2020).

3. Dong, E., Du, H. \& Gardner, L. An interactive web-based dashboard to track COVID-19 in real time. Lancet Infect. Dis. 20, 533-534 (2020).

4. Huang, C. et al. Clinical features of patients infected with 2019 novel coronavirus in Wuhan, China. Lancet 395, 497-506 (2020).

5. Gao, Z. et al. A systematic review of asymptomatic infections with COVID-19. J. Microbiol. Immunol. Infect., https://doi.org/10.1016/j.jmii.2020.05.001 (2020).

6. Shang, J. et al. Structural basis of receptor recognition by SARS-CoV-2. Nature $\mathbf{5 8 1}$, 221-224 (2020).

7. Seidah, N. G. \& Chrétien, M. Proprotein and prohormone convertases: a family of subtilases generating diverse bioactive polypeptides. Brain Res. 848, 45-62 (1999).

8. Wrobel, A. G. et al. SARS-CoV-2 and bat RaTG13 spike glycoprotein structures inform on virus evolution and furin-cleavage effects. Nat. Struct. Mol. Biol. 27, 763-767 (2020).

9. Hoffmann, M., Kleine-Weber, H. \& Pöhlmann, S. A multibasic cleavage site in the spike protein of SARS-CoV-2 is essential for infection of human lung cells. Mol. Cell 78, 779-784 (2020).

10. Bestle, D. et al. TMPRSS2 and furin are both essential for proteolytic activation of SARS-CoV-2 in human airway cells. Life Sci. Alliance 3, e202000786 (2020).

11. Lau, S. Y. et al. Attenuated SARS-CoV-2 variants with deletions at the S1/S2 junction. Emerg. Microbes Infect. 9, 837-842 (2020).

12. Klimstra, W. B. et al. SARS-CoV-2 growth, furin-cleavage-site adaptation and neutralization using serum from acutely infected hospitalized COVID-19 patients. J. Gen. Virol. 101, 1156-1169 (2020).

13. Plante, J. A. et al. Spike mutation D614G alters SARS-CoV-2 fitness. Nature, https://doi. org/10.1038/s41586-020-2895-3 (2020).

14. Zhang, L. et al. SARS-CoV-2 spike-protein D614G mutation increases virion spike density and infectivity. Nat. Commun. 11, 6013 (2020).

15. Xie, X. et al. An infectious cDNA clone of SARS-CoV-2. Cell Host Microb. 27, 841-848 (2020).

16. Harcourt, J. et al. Severe acute respiratory syndrome coronavirus 2 from patient with coronavirus disease, United States. Emerg. Infect. Dis. 26, 1266-1273 (2020).

17. Menachery, V. D. et al. Pathogenic influenza viruses and coronaviruses utilize similar and contrasting approaches to control interferon-stimulated gene responses. MBio $\mathbf{5}$, e01174-14 (2014)

18. Matsuyama, S. et al. Enhanced isolation of SARS-CoV-2 by TMPRSS2-expressing cells. Proc. Natl Acad. Sci. USA 117, 7001-7003 (2020).

19. Imai, M. et al. Syrian hamsters as a small animal model for SARS-CoV-2 infection and countermeasure development. Proc. Natl Acad. Sci. USA 117, 16587-16595 (2020).

20. Tostanoski, L. H. et al. Ad26 vaccine protects against SARS-CoV-2 severe clinical disease in hamsters. Nat. Med. 26, 1694-1700 (2020).

21. Bos, R. et al. Ad26 vector-based COVID-19 vaccine encoding a prefusion-stabilized SARS-CoV-2 Spike immunogen induces potent humoral and cellular immune responses. NPJ Vaccines 5, 91 (2020).

22. Winkler, E. S. et al. SARS-CoV-2 infection of human ACE2-transgenic mice causes severe lung inflammation and impaired function. Nat. Immunol. 21, 1327-1335 (2020).

23. Muruato, A. E. et al. A high-throughput neutralizing antibody assay for COVID-19 diagnosis and vaccine evaluation. Nat. Commun. 11, 4059 (2020)

24. Zhu, N. et al. A novel coronavirus from patients with pneumonia in China, 2019. N. Engl. J. Med. 382, 727-733 (2020).

25. Santiana, M. et al. Vesicle-cloaked virus clusters are optimal units for inter-organismal viral transmission. Cell Host Microbe 24, 208-220.e8 (2018).

26. Rivera-Serrano, E. E., González-López, O., Das, A. \& Lemon, S. M. Cellular entry and uncoating of naked and quasi-enveloped human hepatoviruses. eLife 8, e43983 (2019).

27. Gao, Q. et al. Development of an inactivated vaccine candidate for SARS-CoV-2. Science 369, 77-81 (2020).

28. Hansen, J. et al. Studies in humanized mice and convalescent humans yield a SARS-CoV-2 antibody cocktail. Science 369, 1010-1014 (2020).

Publisher's note Springer Nature remains neutral with regard to jurisdictional claims in published maps and institutional affiliations.

(c) The Author(s), under exclusive licence to Springer Nature Limited 2021 


\section{Methods}

No statistical methods were used to predetermine sample size. The experiments were not randomized, and investigators were not blinded to allocation during experiments and outcome assessment.

\section{Viruses and cells}

The recombinant wild-type and mutant SARS-CoV-2 are based on the sequence of USA-WA1/2020 isolate provided by the World Reference Center for Emerging Viruses and Arboviruses (WRCEVA), which was originally obtained from the USA Centers for Disease Control and Prevention (CDC) as previously described ${ }^{16}$. Wild-type and mutant SARS-CoV-2, as well as recombinant mouse-adapted recombinant SARS-CoV ${ }^{16,29}$, were titrated and propagated on Vero E6 cells or Vero E6 cells expressing TMPRSS2 (Sekisui XenoTech), grown in DMEM with $5 \%$ fetal bovine serum and $1 \%$ antibiotic-antimytotic (Gibco). Calu-3 2B4 cells were grown in DMEM with $10 \%$ defined fetal bovine serum, $1 \%$ sodium pyruvate (Gibco) and $1 \%$ antibiotic-antimitotic (Gibco). Standard plaque assays were used for SARS-CoV and SARS-CoV- ${ }^{29,30}$. All experiments involving infectious virus were conducted at the University of Texas Medical Branch (UTMB), Emory University or Washington University in approved biosafety level (BSL) 3 laboratories with routine medical monitoring of staff.

\section{Construction of $\triangle$ PRRA-mutant viruses}

Both wild-type and mutant viruses were derived from the SARS-CoV-2 USA-WA1/2020 infectious clone, as previously described ${ }^{15}$. For $\triangle$ PRRA construction, the mutation was introduced into a subclone puc57-CoV2-F6 by using overlap PCR with primers $\triangle$ PRRA-F (5'-GACTAATTCTCGTAGTGTAGCTAGTCAATCCATC- $3^{\prime}$ ) and $\triangle$ PRRA-R (5'-GACTAGCTACACTACGAGAATTAGTCTGAGTC-3'). The resulted plasmid was validated by restriction enzyme digestion and Sanger sequencing. Thereafter, plasmids containing wild-type and mutant SARS-CoV-2 genome fragments were amplified and digested by restriction enzyme. The SARS-CoV-2 genome fragments were purified and ligated in vitro to assemble the full-length cDNA, according to previously described procedures ${ }^{15}$. In vitro transcription reactions then were performed to synthesize full-length genomic RNA. To recover the viruses, the RNA transcripts were electroporated into Vero E6 cells. The medium from electroporated cells as collected at $40 \mathrm{~h}$ after infection served as seed stock for subsequent experiments. Viral mutants were confirmed by sequence analysis before use. Synthetic construction of $\triangle$ PRRA SARS-CoV-2 was approved by the UTMB Institutional Biosafety Committee.

\section{In vitro infection}

Viral infections in Vero E6 and Calu-32B4 cells were performed as previously described ${ }^{15,31}$. In brief, cells were washed with PBS and inoculated with SARS-CoV or SARS-CoV-2 at an MOI of 0.01 for $60 \mathrm{~min}$ at $37^{\circ} \mathrm{C}$. Following inoculation, cells were washed and fresh medium was added to denote time 0 . Three or more biological replicates were collected at each described time. No blinding was used in any sample collections, nor were samples randomized. Microsoft Excel for Mac 2011 was used to analyse data.

\section{Virion purification and western blotting}

Vero E6 or Calu-3 2B4 cells were infected with wild-type or $\triangle$ PRRAmutant viruses at an MOI of 0.01 . At 24 or $48 \mathrm{~h}$ after infection, the culture medium was collected and clarified by low speed centrifugation. Virus particles in the supernatant were subsequently pelleted by ultracentrifugation through a $20 \%$ sucrose cushion at $26,000 \mathrm{rpm}$ for $3 \mathrm{~h}$ using a Beckman SW28 rotor. Protein lysates were prepared from the pellets using $2 \times$ Laemmli sample buffer (cat. no. 161-073, BioRad). Relative viral protein levels were determined by SDS-PAGE followed by western blot analysis as previously described ${ }^{16,32-34}$. In brief, sucrose-purified
SARS-CoV, SARS-CoV-2 and $\triangle$ PRRA SARS-CoV-2 were inactivated by boiling in Laemmeli buffer. Samples were loaded in equal volumes into 4-20\% Mini-PROTEAN TGX Gels (Biorad no. 4561093) and electrophoresed by SDS-PAGE. Protein was transferred to polyvinylidene difluoride (PVDF) membranes. Membranes were blotted with SARS-CoV S-specific antibodies (Novus Biologicals no. NB100-56578), followed by probing with horseradish peroxidase (HRP)-conjugated anti-rabbit antibody (Cell Signaling Technology no. 7074S). Blots were stripped and reprobed with SARS-CoV N-specific antibodies (provided by S. Makino) and the HRP-conjugated anti-rabbit secondary IgG. In both cases, signal was developed by treating membranes with Clarity Western ECL substrate (Bio-Rad no. 1705060) imaging on a ChemiDoc MP System (Bio-Rad no. 12003154). Densitometry was performed using ImageLab 6.0.1 (Bio-Rad no. 2012931).

\section{Competition assay and real-time PCR}

For competition assays, ratios (50:50, 90:10 and 10:90 wild type: $\triangle$ PRRA) were determined by PFU derived from viral stocks. Vero cells were infected at an MOI of 0.1 (wild type and $\triangle \mathrm{PRRA}$ ) as described in 'In vitro infection'. RNA from cell lysates was collected using Trizol reagent (Invitrogen). RNA was then extracted from Triazol using the Direct-zol RNA Miniprep Plus kit (Zymo Research no. R2072), as per the manufacturer's instruction. Extracted RNA was then converted to cDNA with the iScript cDNA Synthesis kit (BioRad no. 1708891). RT-qPCR was performed with the Luna Universal qPCR Master Mix (New England Biolabs no. M3003) on a CFX Connect instrument (Bio-Rad no. 1855200). For differentiation between wild-type SARS-CoV-2 and $\triangle$ PRRA SARS-CoV-2 genomes in competition experiments, primer 1 (forward: AATGTTTTTCAAACACGTGCAG) and primer 2 (reverse: TACACTACGTG CCCGCCGAGG) were used to detect wild-type genomes only. For detecting total genomes, primer 1 and primer 3 (reverse: GAATTTTCTGCA CCAAGTGACA) were used. Eight-point standard curves $\left(1 \times 10^{1}\right.$ to $1 \times 10^{8}$ copies per $\mu \mathrm{l}$ ) were used to quantify the signal. A primer annealing temperature of $63^{\circ} \mathrm{C}$ was used for all assays.

For detection of viral RNA, the nasal washes and oral swabs of hamsters infected with wild-type SARS-CoV-2 or $\triangle$ PRRA SARS-CoV-2, RNA extraction, $c D N A$ synthesis and RT-qPCR were performed as described in the preceding paragraph. For RT-qPCR, primer 1 and primer 3 were used for all hamster samples.

\section{Deep sequencing analysis}

RNA libraries were prepared with 300 ng of RNA using the Click-Seq protocol, as previously described ${ }^{35}$, using tiled primers cognate to the SARS-COV-2 genome (accession number NC_045512.2) and the TruSeq i7 LT adaptor series and i5 hexamer adaptors containing a $12 \mathrm{~N}$ unique molecular identifier. Libraries were sequenced on the Illumina MiSeq platform with MiSeq Reagent Kit v.2. Raw data were de-multiplexed using TruSeq indexes using the MiSeq Reporter Software. Fastp v.0.12 was used to trim adaptor sequences and low-quality reads $(q<25)$, to remove reads less than $40 \mathrm{nt}$ in length and to copy unique molecular identifier sequences onto the read name. Reads were aligned with bowtie using the -best parameter, allowing for up to two mismatches. The alignment index was generated from a single fasta file, which contained two 600-nt reference sequences spanning the PRRA locus $(23,603-23,616)$ of the wild-type (accession number NC_045512.2) and $\triangle$ PRRA genomes. The alignments were sorted and indexed using Samtools v.1.9 ${ }^{37}$, PCR duplicates were removed using umi_tools ${ }^{38}$. Coverage at each position was determined with the genomecov function in bedtools v.2.25.0 ${ }^{39}$.

\section{Plaque-reduction neutralization test}

Neutralization assays were performed using mNeonGreen SARS-CoV-2 reporter neutralization assay, as previously described ${ }^{23}$.In brief, Vero E6 cells were plated on a black $\mu$ CLEAR flat-bottom 96-well plate (Greiner Bio-one). On the following day, sera or monoclonal antibodies were 
serially diluted from $1 / 20$ with 9 twofold dilutions to the final dilution of $1 / 5,120$ and incubated with $m$ NeonGreen SARS-CoV-2 or $\triangle$ PRRA expressing $\mathrm{mNeonGreen}$ at $37^{\circ} \mathrm{C}$ for $1 \mathrm{~h}$. The virus-serum mixture was transferred to the Vero E6 cell plate with a final MOI of 0.5. After 20 h, Hoechst 33342 Solution (400-fold diluted in Hank's Balanced Salt Solution (Gibco)) was added to stain the cell nucleus, sealed with Breath-Easy sealing membrane (Diversified Biotech), incubated at $37^{\circ} \mathrm{C}$ for 20 min and quantified for $\mathrm{mNeonGreen} \mathrm{fluorescence} \mathrm{on} \mathrm{Cytation}$ 7 (BioTek). The raw images $(2 \times 2$ montage) were acquired using a $4 \times$ objective, processed and stitched using the default setting. The total cells (indicated by nucleus staining) and mNeonGreen-positive cells were quantified for each well. Infection rates were determined by dividing the mNeonGreen-positive cell number by the total cell number. Relative infection rates were obtained by normalizing the infection rates of serum-treated groups to those of non-serum-treated controls. The curves of the relative infection rates versus the serum dilutions ( $\log _{10}$-transformed values) were plotted using Prism 8 (GraphPad). A nonlinear regression method was used to determine the dilution fold that neutralized $50 \%$ of $\mathrm{mNeonGreen} \mathrm{fluorescence}\left(\mathrm{NT}_{50}\right)$. Each serum was tested in duplicates.

\section{Phylogenetic tree, sequence identity heat map and structural modelling}

Heat maps were constructed from a set of representative group-2B coronaviruses using alignment data paired with neighbour-joining phylogenetic trees built in Geneious (v.9.1.5), using the $S$ amino acid sequences derived the following accession numbers: QHU79204 (SARS-CoV-2 WA1), QHR63300.2 (RATG13), QND76034.1(HKU3), AGZ48828.1(WIV1), AGZ48806 (RsSHC014), ALK02457 (WIV16) and AYV99817.1(SARS-CoV Urbani). Sequence identity was visualized using EvolView (http://www. evolgenius.info/) and SARS-CoV Co-V-2 WA1 served as the reference sequence. Structural models were generated using SWISS-Model ${ }^{40,41}$ to generate homology models for SARS-CoV-2S protein with and without the furin cleavage site on the basis of the SARS-CoV-1 trimer structure (Protein Data Bank code 6ACD). Homology models were visualized and manipulated in MacPyMol (version 1.3).

\section{Transmission electron microscopy}

Supernatants of SARS-CoV-2 infected cells were centrifuged for $10 \mathrm{~min}$ at 3,000 $g$ to remove large cellular debris. Nickel grids were incubated with clarified supernatants for $10 \mathrm{~min}$ followed by glutaraldehyde fixation and $2 \%$ uranyl acetate staining. Micrographs were taken using a JEM 14000 (JEOL USA). Several randomly selected fields were imaged to obtain unbiased particle counts.

\section{Hamster infection studies}

Male Syrian hamsters (7-8 weeks old, $86-127 \mathrm{~g}$ ) were purchased from Envigo. All procedures were conducted under an animal protocol approved by the UTMB Institutional Animal Care and Use Committee and complied with USDA guidelines in a laboratory accredited by the Association for Assessment and Accreditation of Laboratory Animal Care. Work with infectious SARS-CoV-2 in hamsters was performed in the Galveston National Laboratory BSL-4 laboratory. Hamsters were housed in microisolator caging equipped with HEPA filters in the BSL-4 laboratories. Hamsters were challenged with $10^{5} \mathrm{PFU}$ of wild-type or $\triangle$ PRRA SARS-CoV-2 by intranasal inoculation. Hamsters were observed daily for the development of clinical disease and body weights were taken every day for the first 10 days of the study, then every third day. For each manipulation (viral infection, retro-orbital bleeds, nasal wash or oral swab), hamsters were anaesthetized with isoflurane (Piramal).

\section{Mouse infection studies}

Mouse studies were carried out in accordance with the recommendations in the Guide for the Care and Use of Laboratory Animals of the National Institutes of Health. The protocols were approved by the
Institutional Animal Care and Use Committee at the Washington University School of Medicine (assurance number A3381-01) and performed in an ABSL-3 facility. Heterozygous K18-hACE C57BL/6J mice (strain $2 \mathrm{~B} 6$. $\mathrm{Cg}-\mathrm{Tg}(\mathrm{K} 18-\mathrm{ACE} 2) 2 \mathrm{Prlmn} / \mathrm{J}$ ) were obtained from The Jackson Laboratory and randomized to upon arrival. Mice were housed in $7.5 \times 11.5 \times 5^{\prime \prime}$ cages in groups of $\leq 5$ and fed standard chow diets (PicoLab Rodent Diet 5053, Purina). Cages were changed weekly. The ABSL-3 room was kept between 20.0 and $23.3^{\circ} \mathrm{C}$ with $30-60 \%$ and 12 -h-12-h light-dark cycles (06:00 to 18:00 h). Mice of both sexes were used for experimentation. Mice were housed in groups of $\leq 5$ individuals in rooms maintained between 20.0 and $23.3^{\circ} \mathrm{C}$ with $30-60 \%$ humidity. Mice were given ad libitum access to water and PicoLab Rodent Diet 5053 chow (Purina). Virus inoculations were performed under anaesthesia that was induced and maintained with ketamine hydrochloride and xylazine; all efforts were made to minimize the suffering of the mice. Mice of different ages (5-9 weeks old) and both sexes were administered $10^{3}$ PFU of SARS-CoV-2 in a $50-\mu \mathrm{l}$ intranasal dose.

\section{Cytokine and chemokine protein measurements}

Lung homogenates were incubated with Triton-X-100 (1\% final concentration) for $1 \mathrm{~h}$ at room temperature to inactivate SARS-CoV-2. Homogenates then were analysed for cytokines and chemokines by Eve Technologies, using their Mouse Cytokine Array and Chemokine Array 31-Plex (MD31) platform.

\section{Respiratory mechanics}

Mice were anaesthetized with ketamine and xylazine $\left(100 \mathrm{mg} \mathrm{kg}^{-1}\right.$ and $10 \mathrm{mg} \mathrm{kg}^{-1}$ intraperitoneally, respectively). The trachea was isolated via dissection of the neck area and cannulated using an 18-gauge blunt metal cannula (typical resistance of $0.18 \mathrm{cmH}_{2} \mathrm{O}$ per $\mathrm{ml}$ ), which was secured in place with a nylon suture. The mouse then was connected to the Flexivent computer-controlled piston ventilator (SCIREQ) via the cannula, which was attached to the FX adaptor Y-tubing. Mechanical ventilation was initiated, and mice were given an additional $100 \mathrm{mg} \mathrm{kg}^{-1}$ of ketamine and $0.1 \mathrm{mg}$ per mouse of the paralytic pancuronium bromide via the intraperitoneal route to prevent breathing efforts against the ventilator and during measurements. Mice were ventilated using default settings for mice, which consisted of positive-end expiratory pressure at $3 \mathrm{cmH}_{2} \mathrm{O}, \mathrm{a} 10 \mathrm{ml} \mathrm{kg}^{-1}$ tidal volume, a respiratory rate of 150 breaths per minute and a fraction of inspired oxygen of 0.21 (that is, room air). Respiratory mechanics were assessed using the forced oscillation technique, as previously described ${ }^{42}$, using the latest version of the Flexivent operating software (FlexiWare v.8.1.3). Pressure-volume loops and measurements of inspiratory capacity were also performed.

\section{Measurement of viral burden}

Mouse tissues were weighed and homogenized with zirconia beads in a MagNA Lyser instrument (Roche Life Science) in 1,000 $\mu$ l of DMEM supplemented with $2 \%$ heat-inactivated FBS. Tissue homogenates were clarified by centrifugation at 10,000 rpm for $3 \mathrm{~min}$ and RNA was extracted from $50 \mu \mathrm{l}$ of supernatant using the MagMax mirVana Total RNA isolation kit (Thermo Scientific) on the Kingfisher Flex extraction robot (Thermo Scientific). RNA was reverse-transcribed and amplified using the TaqMan RNA-to-CT 1-Step Kit (ThermoFisher). Reverse transcription was carried out at $48^{\circ} \mathrm{C}$ for $15 \mathrm{~min}$ followed by $2 \mathrm{~min}$ at $95^{\circ} \mathrm{C}$. Amplification was accomplished over 50 cycles as follows: $95^{\circ} \mathrm{C}$ for $15 \mathrm{~s}$ and $60^{\circ} \mathrm{C}$ for $1 \mathrm{~min}$. Copies of SARS-CoV-2 $\mathrm{N}$ gene RNA in samples were determined using a previously published assay ${ }^{22}$. In brief, a TaqMan assay was designed to target a highly conserved region of the $N$ gene (forward primer: ATGCTGCAATCGTGCTACAA; Reverse primer:GACTGCCGCCTCTGCTC; probe:/56-FAM/TCAAGGAAC/ZEN/ AACATTGCCAA/3IABkFQ/). This region was included in an RNA standard to allow for copy-number determination down to ten copies per reaction. The reaction mixture contained final concentrations of primers and probe of 500 and $100 \mathrm{nM}$, respectively. 


\section{Histology and RNA in situ hybridization}

Upon euthanasia, the lung was inflated with about $1.2 \mathrm{ml}$ of $10 \%$ neutral buffered formalin using a 3-ml syringe and catheter inserted into the trachea. The airway, lungs and heart were removed en bloc and transferred to a conical flask containing $40 \mathrm{ml} 10 \%$ neutral buffered formalin, in which the tissues were allowed to fix for suspension of neutral buffered formalin for $\geq 7$ days. Tissues were embedded in paraffin, and sections were stained with haematoxylin and eosin by the Washington University Lung Morphology Core. Images were captured using the Nanozoomer (Hamamatsu) at the Alafi Neuroimaging Core at Washington University.

\section{Biological materials}

The recombinant wild-type and mutant SARS-CoV-2 described in this Article are available through the WRCEVA at UTMB through a material transfer agreement.

\section{Reporting summary}

Further information on research design is available in the Nature Research Reporting Summary linked to this paper.

\section{Data availability}

The raw data that support the findings of this study are available from the corresponding author upon reasonable request. Source data are provided with this paper.

29. Roberts, A. et al. A mouse-adapted SARS-coronavirus causes disease and mortality in BALB/c mice. PLoS Pathog. 3, e5 (2007).

30. Sims, A . C et al. Release of severe acute respiratory syndrome coronavirus nuclear import block enhances host transcription in human lung cells. J. Virol. 87, 3885-3902 (2013).

31. Josset, L. et al. Cell host response to infection with novel human coronavirus EMC predicts potential antivirals and important differences with SARS coronavirus. MBio 4 e00165-13 (2013).

32. Sheahan, T., Rockx, B., Donaldson, E., Corti, D. \& Baric, R. Pathways of cross-species transmission of synthetically reconstructed zoonotic severe acute respiratory syndrome coronavirus. J. Virol. 82, 8721-8732 (2008).

33. Menachery, V. D. et al. Attenuation and restoration of severe acute respiratory syndrome coronavirus mutant lacking 2'-O-methyltransferase activity. J. Virol. 88, 4251-4264 (2014).

34. van Tol, S. et al. VAMP8 contributes to the TRIM6-mediated type I interferon antiviral response during West Nile virus infection. J. Virol. 94, 94 (2020).

35. Routh, A., Head, S. R., Ordoukhanian, P. \& Johnson, J. E. ClickSeq: fragmentation-free next-generation sequencing via click ligation of adaptors to stochastically terminated 3'-azido cDNAs. J. Mol. Biol. 427, 2610-2616 (2015).
36. Chen, S., Zhou, Y., Chen, Y. \& Gu, J. fastp: an ultra-fast all-in-one FASTQ preprocessor. Bioinformatics 34, i884-i890 (2018).

37. Li, H. et al. The Sequence Alignment/Map format and SAMtools. Bioinformatics 25, 2078-2079 (2009).

38. Smith, T., Heger, A. \& Sudbery, I. UMI-tools: modeling sequencing errors in unique molecular identifiers to improve quantification accuracy. Genome Res. 27, 491-499 (2017).

39. Quinlan, A. R. \& Hall, I. M. BEDTools: a flexible suite of utilities for comparing genomic features. Bioinformatics 26, 841-842 (2010).

40. Waterhouse, A. et al. SWISS-MODEL: homology modelling of protein structures and complexes. Nucleic Acids Res. 46, W296-W303 (2018).

41. Bienert, S. et al. The SWISS-MODEL repository-new features and functionality. Nucleic Acids Res. 45, D313-D319 (2017).

42. McGovern, T. K., Robichaud, A., Fereydoonzad, L., Schuessler, T. F. \& Martin, J. G. Evaluation of respiratory system mechanics in mice using the forced oscillation technique. J. Vis. Exp. 75, e50172 (2013).

Acknowledgements Research was supported by grants from NIA and NIAID of the NIH (Al153602 and AG049042 to V.D.M.; Al142759, Al134907, Al145617 and UL1TR001439 to P.-Y.S.; R01Al123449 to A.F. and B.L.; R24Al120942 (WRCEVA) to S.C.W.; and R01Al157155 and Defense Advanced Research Project Agency (HR001117S0019) to M.S.D.). Research was also supported by STARs Award provided by the University of Texas System to V.D.M. and trainee funding provided by the McLaughlin Fellowship Fund at UTMB. P.-Y.S. was also supported by a CDC grant for the Western Gulf Center of Excellence for Vector-Borne Diseases, and awards from the Sealy \& Smith Foundation, Kleberg Foundation, John S. Dunn Foundation, Amon G. Carter Foundation, Gilson Longenbaugh Foundation and Summerfield Robert Foundation.

Author contributions X.X., B.A.J., A.L.R., M.S.S., M.S.D., A.N.F., P.-Y.S. and V.D.M conceptualized the project: B.A.J X.X, B.K, K.G.L, D.S. A.L.R. A.N.F., P.Y.S. and V.D.M. developed the methodology; B.A.J., X.X., B.K., K.G.L., A.M., J.Z., X.Z., T.J., J.K.S., L.Z., C.S., M.V., A.V., E.S.W., D.S., N.B., J.A.P., A.L.R., K.D. and V.D.M. performed the investigations; K.S.P., S.C.W., M.S.S., P.R., V.P., Z.K., Z.A., P.-Y.S., M.S.D., A.N.F. and V.D.M. provided resources; B.A.J., X.X., B.K., K.G.L., A.V., E.S.W., D.S., A.L.R., M.S.S., K.D., P.-Y.S., A.N.F. and V.D.M. curated data V.D.M. wrote the original draft; B.A.J., X.X., B.L., P.A., M.S.S., K.D., V.P., Z.K., Z.A., P.-Y.S., A.N.F., V.D.M., M.S.D. and P.-Y.S. reviewed and edited the paper; X.X., B.A.J. A.L.B.,B.K. K.G.L., N.B., A.N.F. and V.D.M. developed the data visualization; P.A., S.C.W., M.S.S., P.-Y.S., A.N.F. and V.D.M. supervised the project; and P.A., S.C.W., P.Y.S., A.N.F. and V.D.M. acquired funding.

Competing interests X.X., P.-Y.S. and V.D.M. have filed a US patent on the reverse genetic system and SARS-CoV-2 reporter. M.S.D. is a consultant for Inbios, Vir Biotechnology and NGM Biopharmaceuticals, and is on the Scientific Advisory Board of Moderna and Immunome. The laboratory of M.S.D. has received unrelated funding support in sponsored research agreements from Moderna, Vir Biotechnology and Emergent BioSolutions. The other authors declare no competing interests.

\section{Additional information}

Supplementary information The online version contains supplementary material available at https://doi.org/10.1038/s41586-021-03237-4

Correspondence and requests for materials should be addressed to V.D.M.

Peer review information Nature thanks the anonymous reviewer(s) for their contribution to the peer review of this work.

Reprints and permissions information is available at http://www.nature.com/reprints. 


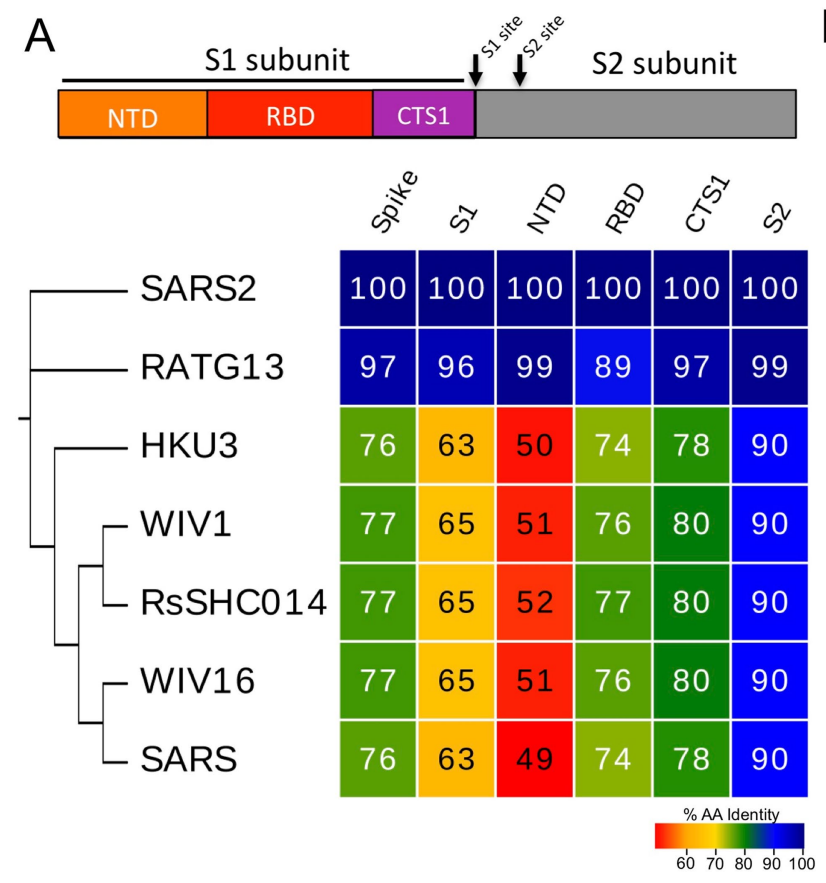

Extended Data Fig.1 | Furin cleavage site in SARS-CoV-2S. a, Diagram of the coronavirus $S$ protein domains and cleavage sites. The sequences of the indicated group-2B coronaviruses were aligned according to the bounds of total S, S1, N-terminal domain (NTD), receptor-binding domain (RBD),

$\mathrm{C}$ terminus of S1 (CTS1) and S2. We extracted sequence identities from the alignments, and constructed a heat map of sequence identity using EvolView

\begin{tabular}{lllllllllllll|l|l|l|llll} 
B & SARS-2 & S & Y & Q & T & Q & T & N & S & P & R & R & A & R & S & V \\
RATG13 & S & Y & Q & T & Q & T & N & S & - & - & - & - & R & S & V \\
HKU3 & S & Y & H & T & A & S & V & L & - & - & - & - & R & S & T \\
WIV1 & S & Y & H & T & V & S & S & L & - & - & - & - & R & S & T \\
SHC014 & S & Y & H & T & V & S & S & L & - & - & - & - & R & S & T \\
WIV16 & S & Y & H & T & V & S & S & L & - & - & - & - & R & S & T \\
SARS & S & Y & H & T & V & S & S & L & - & - & - & - & R & S & T
\end{tabular}
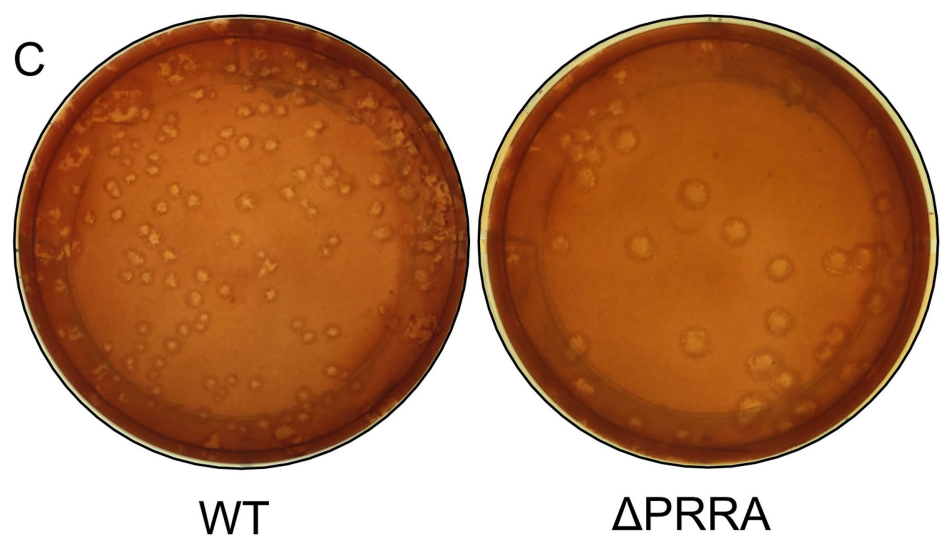

(https://www.evolgenius.info/evolview/) with SARS-CoV-2 WA1 as the reference sequence. $\mathbf{b}$, Alignment of the furin cleavage site of SARS-CoV-2 and the corresponding amino acids identities found in closely related group-2B coronaviruses. The PRRA insertion is unique to SARS-CoV-2. c, Representative plaque morphology of wild-type and $\triangle$ PRRA SARS-CoV-2. 


\section{Article}

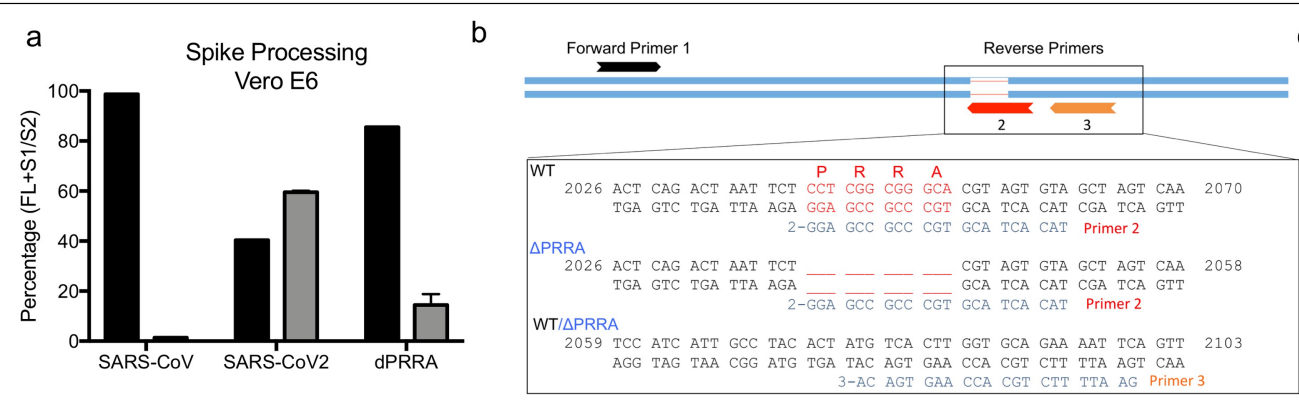

C

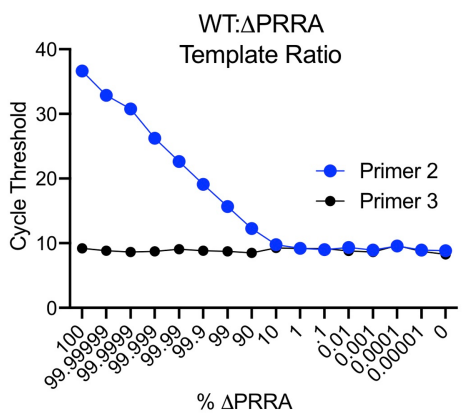

d

$1: 1$

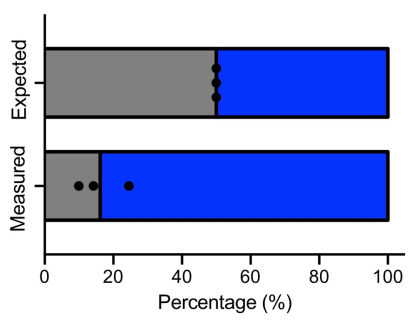

f

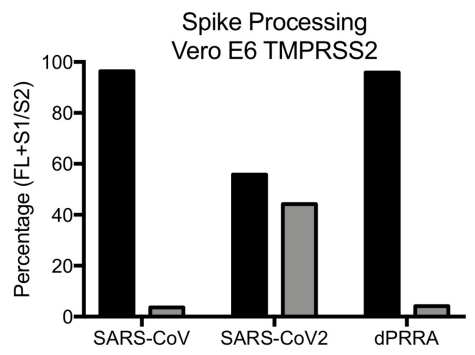

Extended Data Fig. $2 \mid \Delta$ PRRA mutant processing and competition with wild-type SARS-CoV-2.a, Quantification by densitometry of the full-length $\mathrm{S}$ (black) and S1/S2 cleavage form (grey) from western blot experiments in Vero E6 cells $(n=2)$. b, Schematic of RT-qPCR approach to detect deletion of the furin cleavage site. c, Primer curve validation with mixed wild-type/ $\triangle$ PRRA plasmid ratio showing sensitivity. d, Deep sequencing results from $\triangle P R R A$ and wild-type competition assays based on percentage of total reads in that region $(n=3)$.e, Quantification by densitometry of the full-length S (black) and S1/S2 cleavage form (grey) from western blot experiments from Calu-3 cells $(n=2)$. f, Quantification by densitometry of the full-length S (black) and S1/S2 cleavage form (grey) from western blot experiments from Vero E6 cells expressing TMPRSS2 $(n=2)$. Data are mean. 


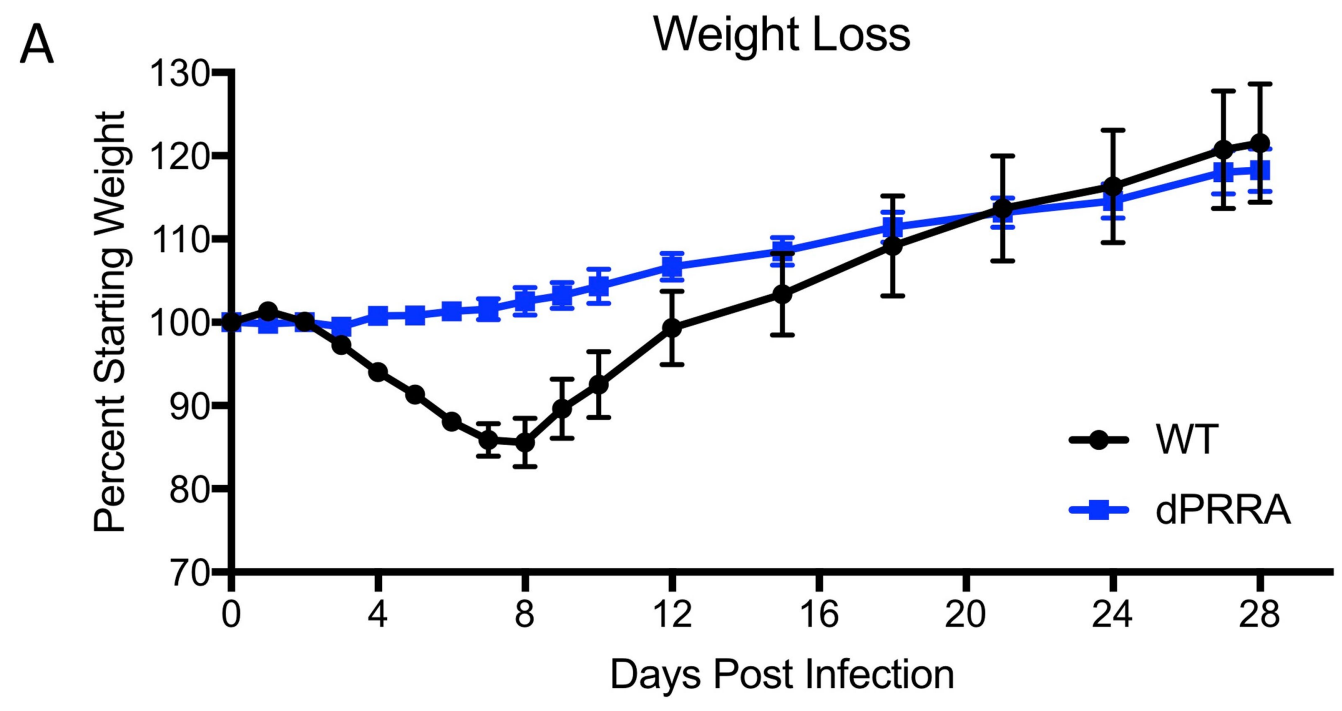

B

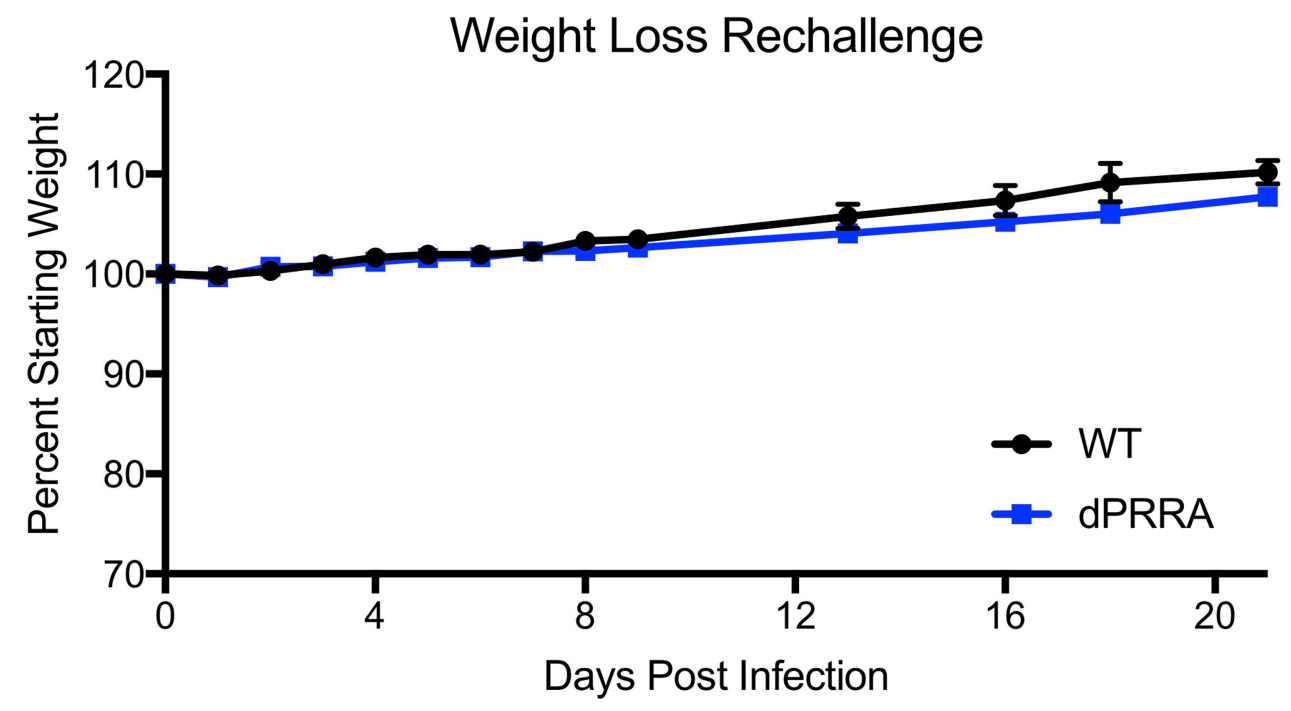

C

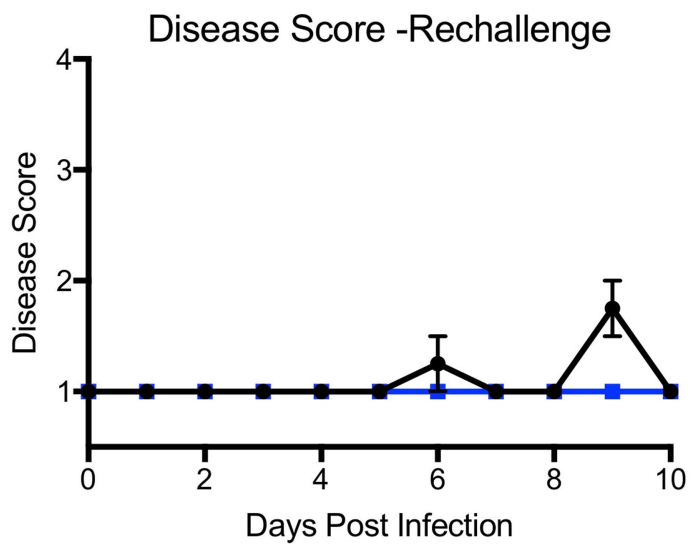

Extended Data Fig. 3 | In vivo attenuation of $\triangle$ PRRA-mutant SARS-CoV-2. a, Weight loss following primary challenge with wild-type or $\triangle P R R A$ SARS-CoV-2 ( $n=4$ hamsters per group). b, c, Weight loss (b) and disease score (c) after rechallenge with wild-type SARS-CoV-2 of hamsters previously infected with wild-type and $\triangle$ PRRA SARS-CoV-2 ( $n=4$ hamsters per group). Data are mean \pm s.e.m. 
a

a
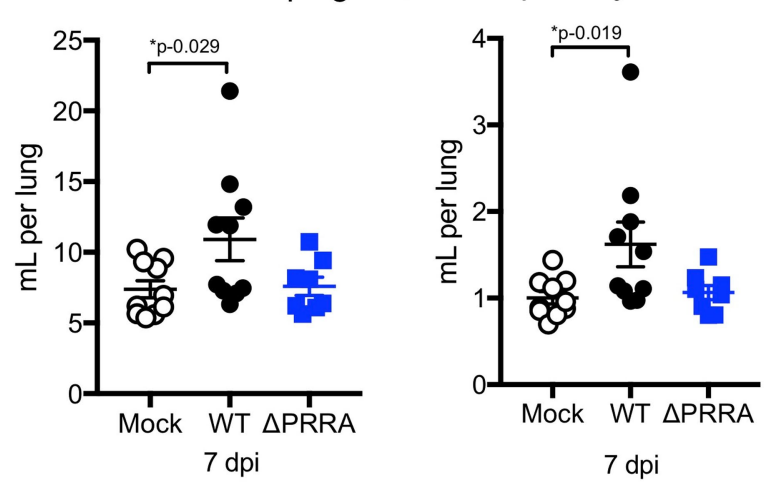

e

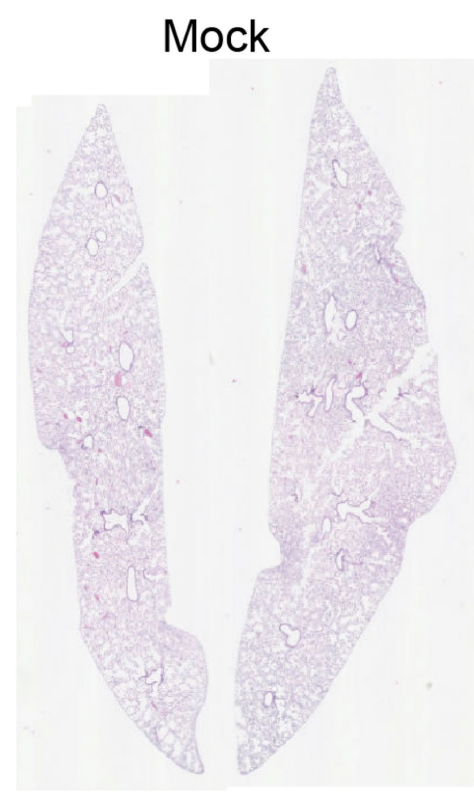

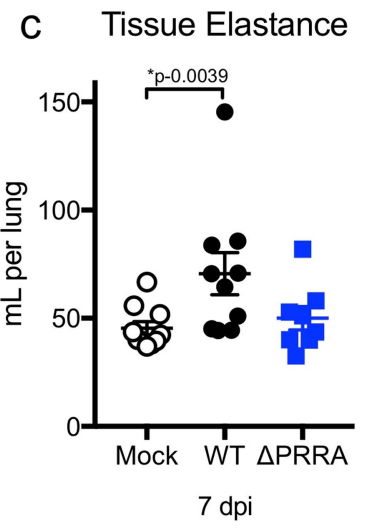

WT

d Newtonian Resistance

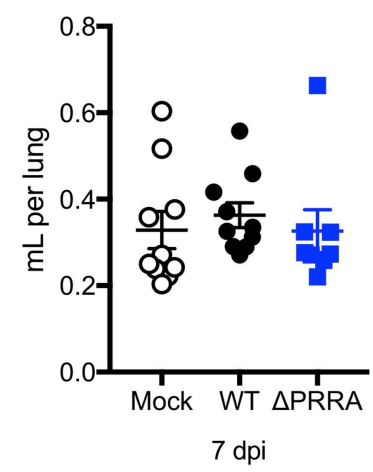

$\triangle \mathrm{PRRA}$

f

GM-CSF

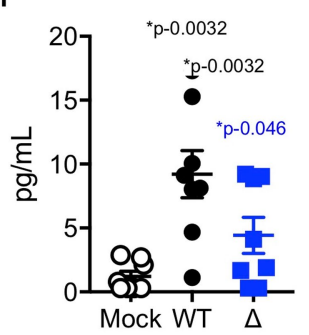

IFN-gamma

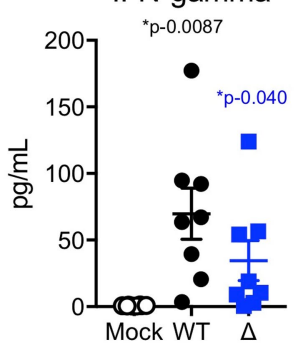

IL-1 $\beta$

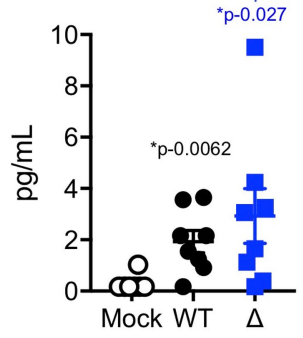

RANTES
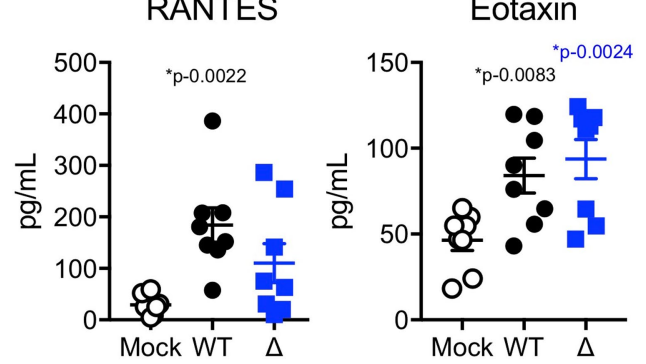

IL-17

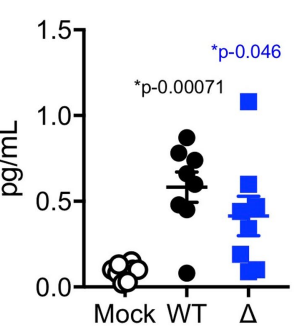

Eotaxin
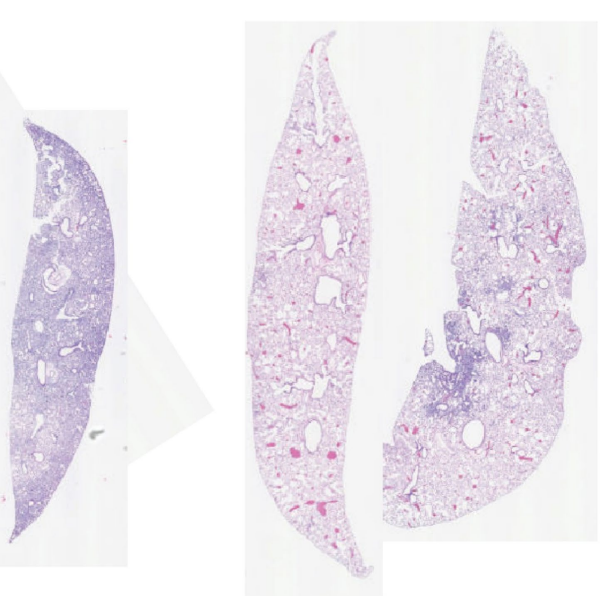

$5 \mathrm{~mm}$

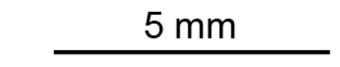

IL-6

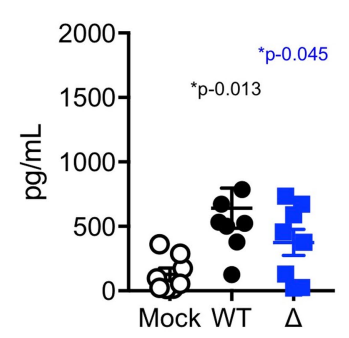

IL-5

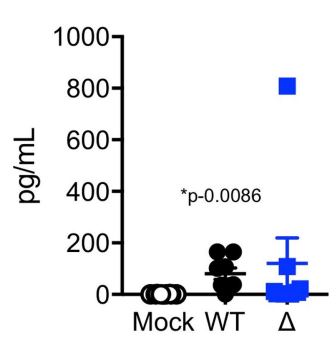

$\mathrm{KC}$

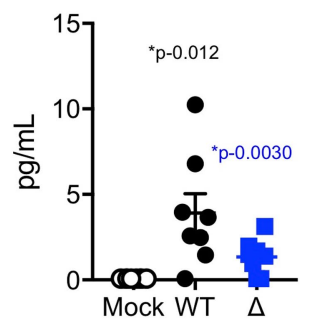

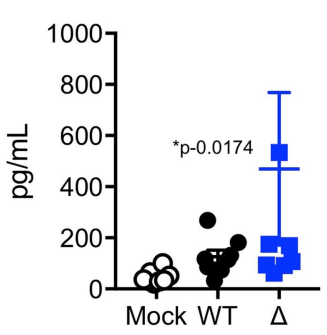

Extended Data Fig. 4 | In vivo attenuation of $\triangle$ PRRA-mutant SARS-CoV-2 in K18-hACE2 mice. a-d, Lung function evaluated using Flexivent for tissue damping (a), respiratory resistance (b), tissue elastance (c) and Newtonian resistance (d). In a-d, $n=10$ mice infected with wild-type SARS-CoV-2, and 8 mice infected with $\triangle$ PRRA SARS-CoV-2.e, Whole lung histopathology sections at $7 \mathrm{dpi}$, from mock-infected mice (i), or mice infected with wild-type (j) or $\triangle$ PRRA (j) SARS-CoV-2, with the least (left) and most (right) severe sections as representative samples from 3 mock-infected, 2 wild-type-infected and $3 \triangle$ PRRA-infected mice. f, Chemokine and cy tokine analysis of mouse lung at 7 days after mock infection (white), or at 7 dpi with wild-type (black) or $\triangle$ PRRA SARS-CoV-2. $n=8$ mice for all groups. $P$ values from two-tailed MannWhitney $(\mathbf{a}-\mathbf{d})$ or two-tailed Student's $t$-tests $(\mathbf{f})$, relative to control. Data are mean \pm s.e.m. Scale bar, $5 \mathrm{~mm}$. 


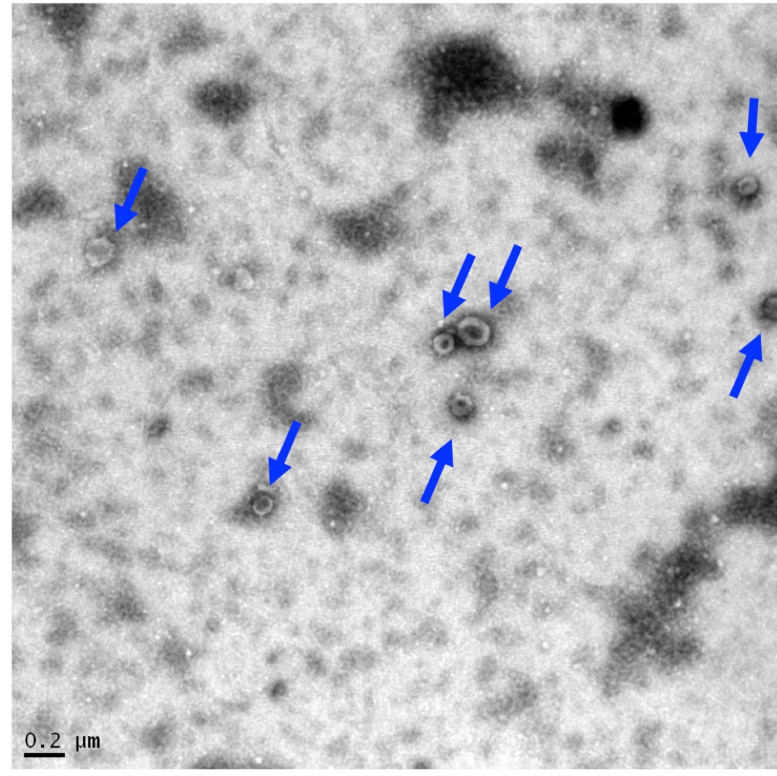

SARS-CoV-2 WT

Extended Data Fig. 5 | Morphology and clumping of $\triangle$ PRRA SARS-CoV-2 virions. Transmission electron microscopy of wild-type (left) and $\triangle P R R A$ (right) SARS-CoV-2 with arrows indicating individual virion particles. Images

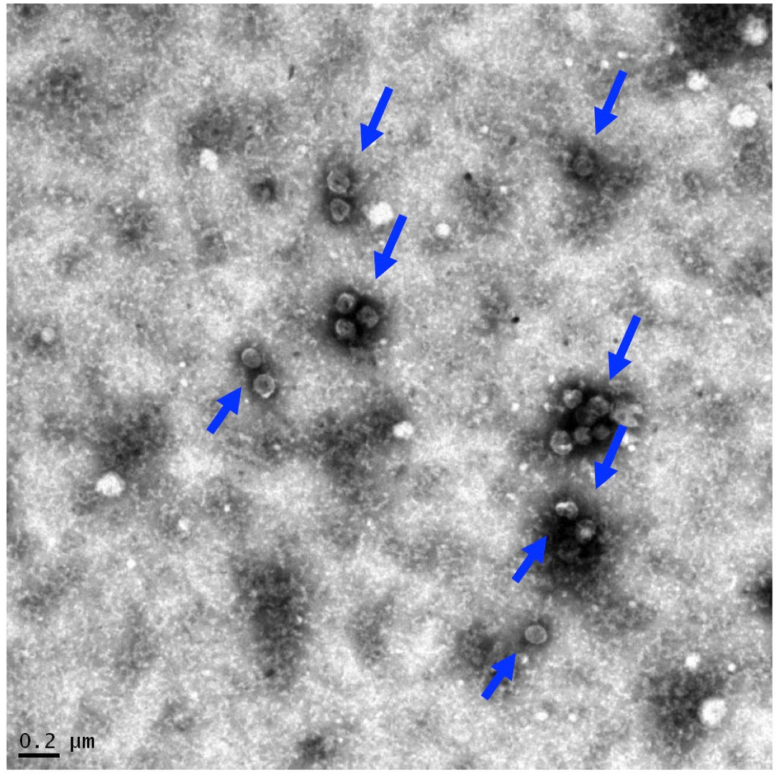

\section{SARS-CoV-2 $\triangle P R R A$}

representative of 2 preparations and 40 individual images observed for wild-type and $\Delta$ PRRA SARS-CoV-2. Scale bars, $0.2 \mu \mathrm{m}$. 


\section{Reporting Summary}

Nature Research wishes to improve the reproducibility of the work that we publish. This form provides structure for consistency and transparency in reporting. For further information on Nature Research policies, see our Editorial Policies and the Editorial Policy Checklist.

\section{Statistics}

For all statistical analyses, confirm that the following items are present in the figure legend, table legend, main text, or Methods section.

$\mathrm{n} / \mathrm{a} \mid$ Confirmed

$\square$ The exact sample size $(n)$ for each experimental group/condition, given as a discrete number and unit of measurement

$\square$ A statement on whether measurements were taken from distinct samples or whether the same sample was measured repeatedly

$\square$ The statistical test(s) used AND whether they are one- or two-sided

Only common tests should be described solely by name; describe more complex techniques in the Methods section.

$\bigotimes \square$ A description of all covariates tested

$\bigotimes \square$ A description of any assumptions or corrections, such as tests of normality and adjustment for multiple comparisons

$\triangle$ A full description of the statistical parameters including central tendency (e.g. means) or other basic estimates (e.g. regression coefficient)

AND variation (e.g. standard deviation) or associated estimates of uncertainty (e.g. confidence intervals)

$\square$ For null hypothesis testing, the test statistic (e.g. $F, t, r$ ) with confidence intervals, effect sizes, degrees of freedom and $P$ value noted

Give $P$ values as exact values whenever suitable.

$\bigotimes \square$ For Bayesian analysis, information on the choice of priors and Markov chain Monte Carlo settings

$\bigotimes \square$ For hierarchical and complex designs, identification of the appropriate level for tests and full reporting of outcomes

$\bigotimes \square$ Estimates of effect sizes (e.g. Cohen's $d$, Pearson's $r$ ), indicating how they were calculated

Our web collection on statistics for biologists contains articles on many of the points above.

\section{Software and code}

Policy information about availability of computer code

Data collection FlexiWare v 8.1.3, Samtools v 1.9., Fastp v0.12, Nanozoomer, SWISS-Model, MacPyMol v 1.3.

Data analysis Data analysis conducted using PRISM 7.0 or Microsoft Excel.

For manuscripts utilizing custom algorithms or software that are central to the research but not yet described in published literature, software must be made available to editors and reviewers. We strongly encourage code deposition in a community repository (e.g. GitHub). See the Nature Research guidelines for submitting code \& software for further information.

\section{Data}

Policy information about availability of data

All manuscripts must include a data availability statement. This statement should provide the following information, where applicable:

- Accession codes, unique identifiers, or web links for publicly available datasets

- A list of figures that have associated raw data

- A description of any restrictions on data availability

The raw data that support the findings of this study are available from the corresponding author upon reasonable request. Spike modeling based on PDB 6ACD. 


\section{Field-specific reporting}

Please select the one below that is the best fit for your research. If you are not sure, read the appropriate sections before making your selection.

$\bigotimes$ Life sciences $\quad \square$ Behavioural \& social sciences $\quad \square$ Ecological, evolutionary \& environmental sciences

For a reference copy of the document with all sections, see nature.com/documents/nr-reporting-summary-flat.pdf

\section{Life sciences study design}

All studies must disclose on these points even when the disclosure is negative.

Sample size Sample sizes for experiments were determined by prior studies with coronaviruses that had power to detect changes in titer with a significance level of 0.05 (two-tailed). Gralinski, L.E., et al.mBio 4(2013).

Data exclusions No data was excluded from the cacluation

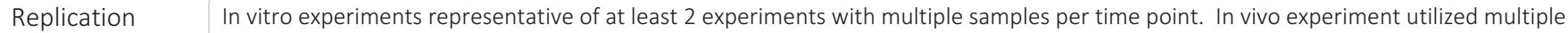
animal per group per time point and were from a single experiment (hamster) and 3 independent experiments (mice).

Randomization Animals were assigned to groups at random. Cell for in vitro experiments were assigned at random.

Blinding Investigators were not blinded during data collection or due to the limited capacity of workers able to complete high containment work. Data analysis was conducted by researcher who was not blinded.

\section{Reporting for specific materials, systems and methods}

We require information from authors about some types of materials, experimental systems and methods used in many studies. Here, indicate whether each material, system or method listed is relevant to your study. If you are not sure if a list item applies to your research, read the appropriate section before selecting a response.

Materials \& experimental systems

$\mathrm{n} / \mathrm{a}$ Involved in the study

\ Antibodies

¿ukaryotic cell lines

Х Palaeontology and archaeology

$\square$ Animals and other organisms

\ $\square$ Human research participants

$\bigotimes \square$ Clinical data

Х

Dual use research of concern

\begin{tabular}{l|l} 
Methods \\
\hline n/a & Involved in the study \\
$\square$ & $\square$ ChIP-seq \\
$\square$ & $\square$ Flow cytometry \\
$\square$ & $\square$ MRI-based neuroimaging
\end{tabular}

\section{Antibodies}

Antibodies used

RabbitSARS-CoV Spike (S) specific antibodies (Novus Biologicals \#NB100-56578)

Horseradish peroxidase (HRP)-conjugated anti-rabbit antibody (Cell Signaling Technology \#7074S)

SARS-CoV Nucleocapsid (N) specific antibodies were generated in house by Dr. Shinji Makino provided as gift.

Validation

Spike antibody- Orthogonal Validation- The target protein is examined with an antibody independent strategy and compared with results from an antibody-dependent strategy. A correlation between these two strategies indicates specificity between the antibody and its target protein. Examples of antibody independent techniques may include in situ hybridization, quantitative PCR, RNA-seq or mass spectrometry. Full length Spike protein transfected into UM92 cells was used as a positive control and an approximate $139 \mathrm{kDa}$ band was observed. Dot Blot results using recombinant proteins for cross-reactivity testing revealed high reactivity to SARS-CoV-2 Spike Protein, 1000-1200 a.a. (NBP2-90973) and low/no reactivity towards MERS Spike 2 or H1N1 (NBP1-99041).

HPR anti-rabit- This product is thoroughly validated with CST primary antibodies and will work optimally with the CST western immunoblotting protocol, ensuring accurate and reproducible results.

SARS-CoV Nucleocapsid (N) specific antibodies (provided as a kind gift from Dr. Shinji Makino) - Previously published Harcourt et ao. Emerg Infect Dis. 2020 Jun;26(6):1266-1273. doi: 10.3201/eid2606.200516. Epub 2020 Jun 17. The plasmid, pBM302, (Das D. and Suresh MR, J Virol Methods. 2006 Nov;137(2):343-6), was used to express SARS-CoV N protein, with a C-terminal His6 tag, to high levels within the inclusion bodies of E.coli and the recombinant protein was purified from the inclusion bodies by nickel-affinity column chromatography under denaturing conditions. The recombinant SARS-CoV N protein was refolded by stepwise dialysis against Tris/phosphate buffer with decreasing concentrations of urea to renature the protein. The renatured, full-length, SARS-CoV N protein was used to immunize rabbits to generate an affinity-purified rabbit anti-SARS-CoV N polyclonal antibody. 


\section{Eukaryotic cell lines}

Policy information about cell lines

Cell line source(s)

VeroE6 (gift from Ralph S. Baric, lab derivative of VERO C1008 ATCC CRL-1586); Calu3 2B4 (gift from Ralph Baric, lab derivative of ATCC HTB-55)

Authentication

None of the cells were authenticated

Mycoplasma contamination

VeroE6 and Calu3 2B4 cells are tested for mycoplasma contamination by RT-PCR periodically. Calu3 cells tested positive for mycoplasma contamination in July. Calu3 and Vero rederived from frozen stocks tested negative in August.

Commonly misidentified lines

(See ICLAC register)

None

\section{Animals and other organisms}

Policy information about studies involving animals; ARRIVE guidelines recommended for reporting animal research

Laboratory animals

Wild animals

Field-collected samples

Ethics oversight
Male Syrian hamsters (7-8 weeks old, 86-127g). Male and female K18-hACE C57BL/6J mice (strain: 2B6.Cg-Tg(K18-ACE2)2Prlmn/J, 5-9 wks old) were obtained from The Jackson Laboratory. The ABSL-3 room was kept between 68-74 degrees Farenheit with 30-60\% and $12 \mathrm{~h}-12 \mathrm{~h}$ light cycles (6AM-6PM)

Study did not involve wild animals

Study did not involve field collected samples

All procedures were conducted under an animal protocol approved by the UTMB Institutional Animal Care and Use Committee and complied with USDA guidelines in an AAALAC-accredited lab. Animal Care and Use Committee at the Washington University School of Medicine (assurance number A3381-01)

Note that full information on the approval of the study protocol must also be provided in the manuscript. 